\title{
Metal-Free Synthesis of Biaryl- and Teraryl-Cored Diarylmethanes by Ring Transformation of $2 \mathrm{H}$-Pyran-2-ones
}

\author{
Fateh V. Singh* (D) \\ Priyanka B. Kole \\ Chemistry Division, School of Advanced Science, \\ VIT University, Chennai Campus, Chennai \\ 600127, Tamil Nadu, India \\ fatehveer.singh@vit.ac.in
}

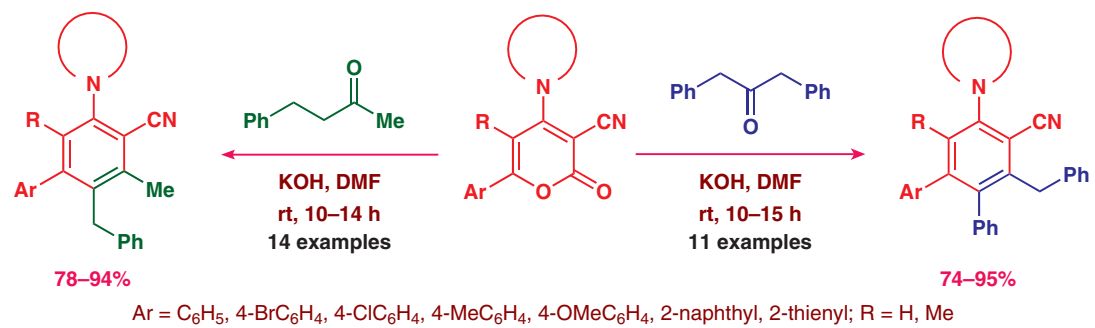

$\mathrm{Ar}=\mathrm{C}_{6} \mathrm{H}_{5}, 4-\mathrm{BrC}_{6} \mathrm{H}_{4}, 4-\mathrm{ClC}_{6} \mathrm{H}_{4}, 4-\mathrm{MeC}_{6} \mathrm{H}_{4}, 4-\mathrm{OMeC}_{6} \mathrm{H}_{4}$, 2-naphthyl, 2-thienyl; $\mathrm{R}=\mathrm{H}, \mathrm{Me}$
Received: 06.09.2018

Accepted after revision: 26.10 .2018

Published online: 30.11 .2018

DOI: 10.1055/s-0037-1610332; Art ID: ss-2018-z0598-op

Abstract An efficient metal-free approach for the synthesis of functionalized biaryl-cored diarylmethanes is described by the ring transformation of $2 \mathrm{H}$-pyran-2-ones using 4-phenylbutan-2-one as carbanion source. Moreover, $2 \mathrm{H}$-pyran-2-ones were reacted with 1,3-diphenylacetone in the presence of base to achieve functionalized teraryl-cored diarylmethanes. All the ring transformation reactions were performed under mild reaction conditions to afford the biaryl- and teraryl-cored reaction products in high yields.

Key words biaryl-cored diarylmethanes, teraryl-cored diarylmethanes, $2 \mathrm{H}$-pyran-2-ones, carbanion, ring transformation reactions, 4phenylbutan-2-one, 1,3-diphenylacetone

Functionalized diarylmethanes are important scaffolds found in various biologically active synthetic and naturally occurring compounds. ${ }^{1}$ Diarylmethane-cored compounds are known to exhibit various biological activities such as antibreast cancer, ${ }^{2}$ thyroid hormone and histamine H1-receptor antagonist, ${ }^{3,4}$ antiviral, ${ }^{5}$ antiallergic, ${ }^{6}$ and antidiabetic activities. ${ }^{7}$ Molecules embedded with diarylmethane units are widely found in various biologically active compounds (Figure 1). Beclobrate (1) has been introduced as potent cholesterol and triglyceride-lowering drug. ${ }^{8}$ Trimethoprim (2) has been developed as effective antibiotic and used for the treatment of urinary tract infections. ${ }^{9}$ In addition, naturally occurring compound avrainvilleol (3) is isolated from red algae and found to exhibit antioxidant activity.$^{10}$ Moreover, the alkaloid papaverine (4), isolated from Papaver somniferum L., exhibits diverse biological activities such as antiplasmodic activity, ${ }^{11}$ cerebral vasodilator, ${ }^{12}$ and non-selective phosphodiesterase inhibitory activity. ${ }^{13}$ In addition, various tetronic acid derived chiral analogues of diarylmethanes have been found to show anti-HIV and an- ticancer activities. ${ }^{14}$ Recently diarylmethane derivatives have been used as an important precursors in several dye preparations. ${ }^{15}$<smiles>CCOC(=O)C(C)(C)Oc1ccc(Cc2ccc(Cl)cc2)cc1</smiles>

1: Beclobrate<smiles>OCc1cc(Br)c(O)c(O)c1Cc1ccc(O)c(Br)c1</smiles>

3: Avrainvilleol<smiles>COc1cc(Cc2cnc(N)nc2N)cc(OC)c1OC</smiles>

2: Trimethoprim<smiles>COc1ccc(Cc2nccc3cc(OC)c(OC)cc23)cc1OC</smiles>

4: Papaverine
Figure 1 Structures of synthetic and natural products of biological importance with diarylmethane units

There are several methods available in literature for the synthesis of diarylmethanes, but most of them are associated with transition-metal-catalyzed coupling reactions such as Pd-catalyzed Suzuki type cross-coupling reactions of benzylic halides with arylboranes. ${ }^{16}$ In 2015, Yoshikai and co-workers reported the synthesis of diarylmethanes by ortho- $\mathrm{CH}$ benzylation of arylimines using Cobalt-Pyphos catalytic system. ${ }^{17}$ In 2016, Zhang and co-workers described a one-pot synthesis of diarylmethanes from benzyl chlorides by a Ni-catalyzed reductive cross-coupling reactions. ${ }^{18}$

Furthermore, synthesis of allyldiarylmethanes was accomplished via 1,6-conjugate allylation of p-quinonemethides using $\mathrm{B}\left(\mathrm{C}_{6} \mathrm{~F}_{5}\right)_{3}$ as catalyst. ${ }^{19}$ In 2016 , Hemelaere and co-workers developed the synthesis of diarylmethanes by Friedel-Crafts reaction of benzyl fluorides in the presence of TFA. ${ }^{20}$ Furthermore, the synthesis of diaryl- 
methanes was achieved through transition-metal-free cross-coupling reaction of benzylic bromides with arylboronic acids in the presence of $\mathrm{Cs}_{2} \mathrm{CO}_{3}$ using the solvent combination $\mathrm{BTF} / \mathrm{H}_{2} \mathrm{O}(10: 1){ }^{21}$

Most of the existing approaches are associated with some limitations such as the use of toxic transition-metal catalysts and harsh reaction conditions. Despite the availability of several existing approaches, there is still scope to develop a new approach that could overcome the problems associated with them and offers the flexibility of introducing a wide range of functional groups in the diarylmethane architecture.

Herein, we report a metal-free approach for the synthesis of functionalized biaryl-cored diarylmethanes $\mathbf{9}$ in high yields by the ring transformation of 6-aryl-2H-pyran-2ones 8 using 4-phenylbutan-2-one (6) as a source of nucleophile. The parent precursors 5 were synthesized by the reaction of methyl 2-cyano-3,3-dimethylsulfanylacrylate with functionalized acetophenones in DMSO at room temperature under alkaline conditions. ${ }^{22}$ Furthermore, the substrates 5 were reacted with secondary amines in methanol at reflux temperature to synthesize 6-aryl-4-amino- $2 \mathrm{H}$ pyran-2-ones $8 .{ }^{22}$

The ring transformation of 2H-pyran-2-ones has been used to synthesize various arenes, ${ }^{23}$ heteroarenes, ${ }^{24}$ and fused cyclic systems. ${ }^{25}$ Recently, we have reported the ultrasound-assisted synthesis of functionalized 2-tetralones via ring transformation of $2 \mathrm{H}$-pyran-2-ones. ${ }^{26}$

Our approach to prepare functionalized biaryl-cored diarylmethanes 9 was based on the ring transformation of 6 aryl-2H-pyran-2-ones $\mathbf{5}$ and $\mathbf{8}$ using 4-phenylbutan-2-one (6) as a carbanion source. Both substrates $\mathbf{5}$ and $\mathbf{8}$ have three electrophilic centers at C-2, C-4, and C-6. The presence of the electron-withdrawing substituent at $\mathrm{C}-3$ position of pyran ring and the extended conjugation makes the latter position to be more reactive towards nucleophiles.

Initially, our studies were focused on the ring transformation of 3-cyano-4-methylsulfanyl-2H-pyran-2-ones (5a) with 4-phenylbutan-2-one (6). The ring transformation of substrate $\mathbf{5 a}$ with $\mathbf{6}$ was performed in DMF in the presence of $\mathrm{KOH}$ at room temperature (Table 1, entry 1 ). Unfortunately, the reaction suffered from several unwanted side reactions and ring transformed product $\mathbf{7 a}$ was obtained in $50 \%$ yield. When other substrates $\mathbf{5 b}$ and $\mathbf{5 c}$ were used in similar reactions and the desired products were observed in slightly improved yields (entries 2 and 3). Probably, the presence of SMe group at C-4 position of lactone ring in substrate $\mathbf{5}$ makes this position more susceptible for nucleophilic attack, which leads to various undesired side products.

Next, our efforts were directed to limit the reactivity of $2 \mathrm{H}$-pyran-2-ones 5 at C-4 position towards the nucleophile. In order to limit the reactivity at C-4 position, the leaving group SMe in substrates $\mathbf{5}$ was replaced with tert-amino functionality by treating with cyclic amines and new sub-
Table 1 Ring Transformation of 6-Aryl-4-(methylthio)-2-oxo- $2 \mathrm{H}$ pyran-3-carbonitriles $\mathbf{5 a - c}$ with 4-Phenylbutan-2-one (6)

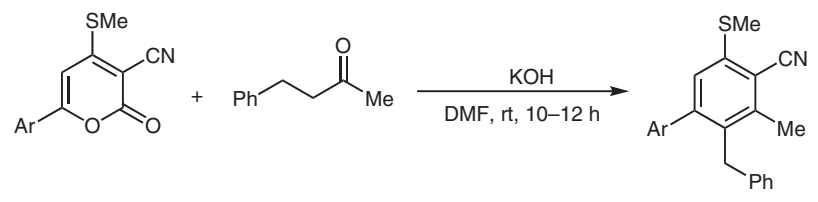

5

6

7: $50-57 \%$

\begin{tabular}{llll}
\hline Entry & $\mathrm{Ar}$ & Reaction time (h) & Yield (\%) of 7 \\
\hline 1 & $\mathrm{Ph}$ & 10 & 50 \\
2 & $4-\mathrm{BrC}_{6} \mathrm{H}_{4}$ & 12 & 52 \\
3 & $4-\mathrm{MeOC}_{6} \mathrm{H}_{4}$ & 10 & 57 \\
\hline
\end{tabular}

strates 6-phenyl-4-amino-2H-pyran-2-ones 8 were synthesized. ${ }^{22}$ Further, to know the influence of amino functionality in the ring transformation reaction, the substrate 2-oxo6-phenyl-4-(piperidin-1-yl)-2H-pyran-3-carbonitrile (8a) was treated with the same ketone $\mathbf{6}$ under similar reaction conditions and a significant improvement was observed in the yield of ring-transformed product $\mathbf{9 a}$ (Scheme 1).

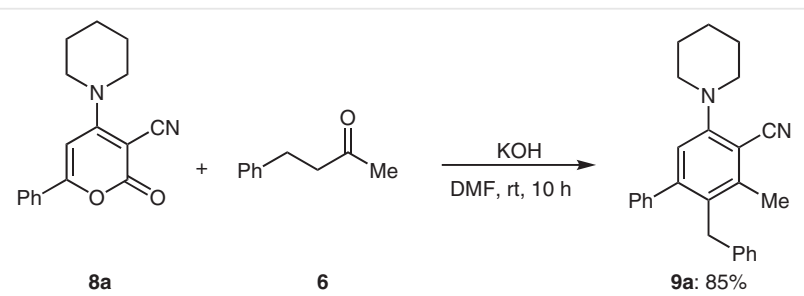

Scheme 1

After that our efforts were directed towards the screening of different solvents using 8a as model substrate. Various polar and nonpolar solvents were employed for the ring transformation of model substrate 8a to the corresponding synthesis of diarylmethane derivative $\mathbf{9 a}$ using ketone $\mathbf{6}$ as source of carbanion and the results obtained are summarized in Table 2. Initially, the ring transformation reaction of 8a was performed in polar and aprotic solvents DMF and DMSO, and the reaction product $9 a$ was isolated in $85 \%$ and $82 \%$ yield, respectively (Table 2, entries 1 and 2). The yield of ring transformed product was slightly lowered when acetonitrile was used as solvent (entry 3 ). The yield was further reduced up to 69\% yield in EtOAc (entry 4). Additional$\mathrm{ly}$, the ring transformation reaction was performed in polar and protic solvents but could not proceed (entries 5-7). The ring transformation reaction could proceed in dichloromethane but desired product 9a was obtained in moderate yield (entry 8). The course of reaction was investigated in aprotic and nonpolar solvents such as benzene and toluene. The ring transformed product was formed in toluene but was observed only in traces in benzene (entries 9 and 10). 
Finally, the reaction was performed in THF, diethyl ether, and 1,4-dioxane but the reaction did not proceed in any of these solvents (entries 11-13).

Table 2 Solvent Optimization for the Ring Transformation of 6-Phenyl$2 \mathrm{H}$-pyran-2-one $8 \mathrm{a}$ to Diarylmethane Derivative 9a

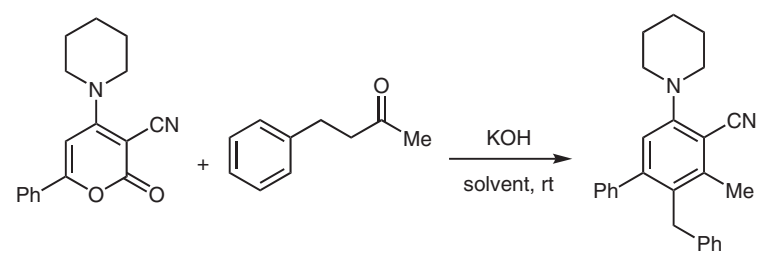

$8 a$

6

9

\begin{tabular}{clll}
\hline Entry & Solvent & Reaction time (h) & 9a Yield (\%) \\
\hline 1 & DMF & 10 & 85 \\
2 & DMSO & 10 & 82 \\
3 & MeCN & 10 & 79 \\
4 & EtOAC & 10 & 69 \\
5 & AcOH & 14 & - \\
6 & MeOH & 14 & - \\
7 & EtOH & 14 & - \\
8 & $\mathrm{CH}_{2} \mathrm{Cl}$ & 12 & 58 \\
9 & toluene & 12 & 30 \\
10 & benzene & 12 & - \\
11 & Et ${ }_{2} \mathrm{O}$ & 12 & - \\
12 & THF & 14 & - \\
13 & $1,4-d i o x a n e$ & 14 & \\
\hline
\end{tabular}

After determining the optimal solvent, our aim was to screen the different bases for the same ring transformation reaction. Several bases were employed for the ring transformation of $\mathbf{8 a}$ to the desired product $\mathbf{9 a}$ and results are listed in Table 3. Initially, the reaction was carried out with $\mathrm{KOH}$ in DMF and ring transformation product was obtained in $85 \%$ yield (Table 3 , entry 1 ). Similarly, the reaction was tested with $\mathrm{NaHCO}_{3}$ under the same reaction condition and the product 9a was isolated in $75 \%$ yield (entry 2 ). Additionally, $\mathrm{K}_{2} \mathrm{CO}_{3}$ and $\mathrm{Cs}_{2} \mathrm{CO}_{3}$ were also used as base for same reaction and desired product $9 \mathrm{a}$ was achieved in $65 \%$ and $60 \%$ yield, respectively (entries 3 and 4). The course of reaction was quite similar with $\mathrm{LiOH}$ and the reaction product 9a was isolated in 63\% yield (entry 5).

After the optimization studies, the presence of $\mathrm{KOH}$ as base and DMF as solvent at room temperature for 10 hours was found as the best reaction conditions for the ring transformation of 6-phenyl-2H-pyran-2-one 8a to 2-benzyl-3methyl-5-(piperidin-1-yl)[1,1'-biphenyl]-4-carbonitrile (9a).

After achieving the best reaction conditions, a series of biaryl-cored diarylmethanes $\mathbf{9 a - k}$ were synthesized in 78$94 \%$ yields by the reaction of various $2 H$-pyran-2-ones $8 \mathbf{a}-\mathbf{k}$
Table 3 Base Optimization for the Ring Transformation of 6-Phenyl$2 \mathrm{H}$-pyran-2-one 8a to Diarylmethane Derivative 9a

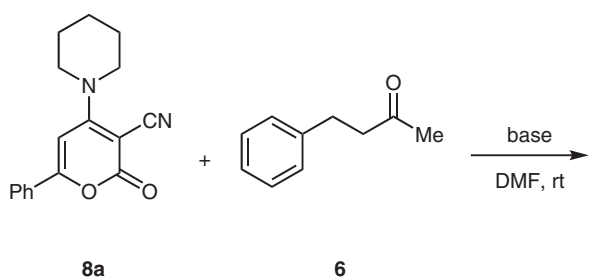<smiles>N#Cc1c(-c2ccccc2)cc(N2CCCCC2)c(C#N)c1[N+](=O)[O-]</smiles>

9a

\begin{tabular}{llll}
\hline Entry & Base & Reaction time (h) & 9a Yield (\%) \\
\hline 1 & $\mathrm{KOH}$ & 10 & 85 \\
2 & $\mathrm{NaHCO}_{3}$ & 12 & 75 \\
3 & $\mathrm{~K}_{2} \mathrm{CO}_{3}$ & 15 & 65 \\
4 & $\mathrm{Cs}_{2} \mathrm{CO}_{3}$ & 15 & 60 \\
5 & $\mathrm{LiOH}$ & 15 & 63 \\
\hline
\end{tabular}

with 4-phenyl-butan-2-one (6) in DMF in the presence of $\mathrm{KOH}$ for 10-14 hours at room temperature (Table 4, entries 1-11). The ring transformation reaction proceeded well with both electron-withdrawing and electron-donating groups on the aromatic ring of 6-aryl-2H-pyran-2-ones 8 . Notably, the ring transformation products $\mathbf{9 f}-\mathbf{h}$ were ob-

Table 4 Synthesis of Biaryl-Cored Diarylmethanes $\mathbf{9 a - k}$ via Ring Transformation of 6-Aryl-2H-pyran-2-ones $\mathbf{8} \mathbf{a}-\mathbf{k}$

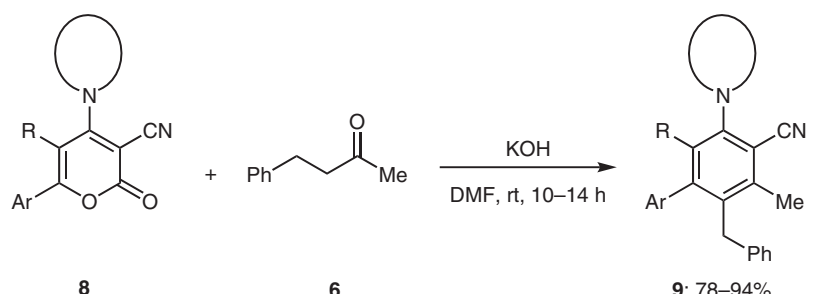

\begin{tabular}{|c|c|c|c|c|c|}
\hline Entry & $\mathrm{Ar}$ & $\mathrm{R}$ & & $\begin{array}{l}\text { Time } \\
\text { (h) }\end{array}$ & $\begin{array}{l}9 \text { Yield } \\
(\%)\end{array}$ \\
\hline 1 & $\mathrm{Ph}$ & $\mathrm{H}$ & piperidin-1-yl & 10 & 85 \\
\hline 2 & $\mathrm{Ph}$ & $\mathrm{H}$ & 4-phenylpiperazin-1-yl & 10 & 82 \\
\hline 3 & $4-\mathrm{ClC}_{6} \mathrm{H}_{4}$ & $\mathrm{H}$ & piperidin-1-yl & 14 & 78 \\
\hline 4 & $4-\mathrm{BrC}_{6} \mathrm{H}_{4}$ & $\mathrm{H}$ & piperidin-1-yl & 12 & 82 \\
\hline 5 & $4-\mathrm{BrC}_{6} \mathrm{H}_{4}$ & $\mathrm{H}$ & 4-phenylpiperazin-1-yl & 12 & 80 \\
\hline 6 & 4- $\mathrm{MeC}_{6} \mathrm{H}_{4}$ & $\mathrm{H}$ & piperidin-1-yl & 10 & 94 \\
\hline 7 & 4- $\mathrm{MeOC}_{6} \mathrm{H}_{4}$ & $\mathrm{H}$ & piperidin-1-yl & 10 & 90 \\
\hline 8 & 4- $\mathrm{MeOC}_{6} \mathrm{H}_{4}$ & $\mathrm{H}$ & 4-phenylpiperazin-1-yl & 10 & 92 \\
\hline 9 & 2-naphthyl & $\mathrm{H}$ & piperidin-1-yl & 10 & 88 \\
\hline 10 & $\mathrm{Ph}$ & Me & 4-phenylpiperazin-1-yl & 12 & 78 \\
\hline 11 & 2-thienyl & $\mathrm{H}$ & piperidin-1-yl & 10 & 83 \\
\hline
\end{tabular}


Table 5 Synthesis of Teraryl-Cored Diarylmethanes $\mathbf{1 1 a - j}$ and $\mathbf{1 1}$ I via Ring Transformation of 6-Aryl-2 $\mathbf{H}$-pyran-2-ones $\mathbf{8 a - j}$ and $\mathbf{8 l}$

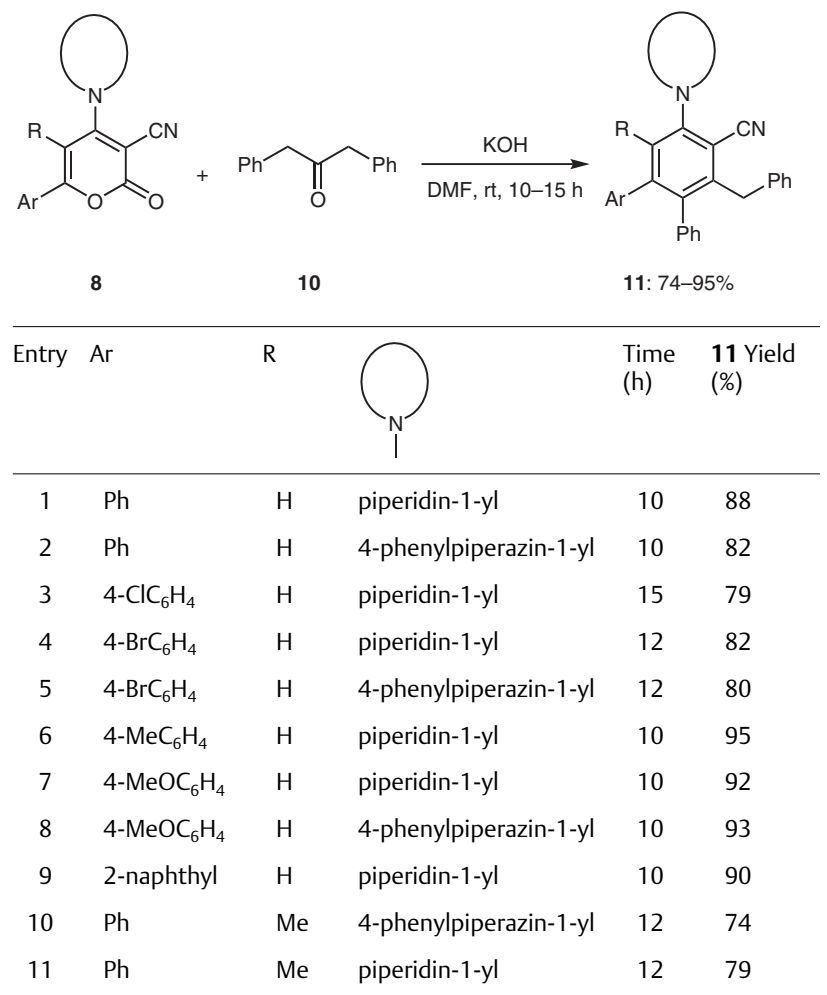

tained in slightly higher yields when the reactions were performed with the substrates $\mathbf{8 f}-\mathbf{h}$ (entries 6-8) having electron-donating groups compared to substrates with electron-withdrawing groups 8c-e (entries 3-5). Furthermore, the ring transformation reaction proceeded well with substrate 8i having naphthyl group at C-6 position and the desired product $9 \mathbf{i}$ was isolated in $88 \%$ yield (entry 9 ). Additionally, the course of reaction was also investigated with the substrate $8 \mathbf{j}$ having methyl group at C-5 position and reaction proceeded well with up to $78 \%$ yield (entry 10 ). The course of reaction was also evaluated with the substrate 8k containing thiophene functionality at C-6 position and reaction worked well with up to $83 \%$ yield (entry 11 ).

In order to generalize this approach, the same synthetic protocol was applied to construct teraryl-cored diarylmethanes 11. To achieve the synthesis of teraryl-cored diarylmethanes 11, the similar substrates 6-aryl-2H-pyran-2ones 8 were treated with 1,3-diphenylacetone (10) in DMF in the presence of $\mathrm{KOH}$ at room temperature and terarylcored diarylmethanes 11 were obtained $74-95 \%$ yields (Table 5, entries 1-11). Various functional groups were successfully tolerated during these ring transformations.

It was observed that the course of reaction was quite similar with both electron-donating and -withdrawing cored substrates but ring transformation products were obtained in slightly higher yields in the case of substrates hav-

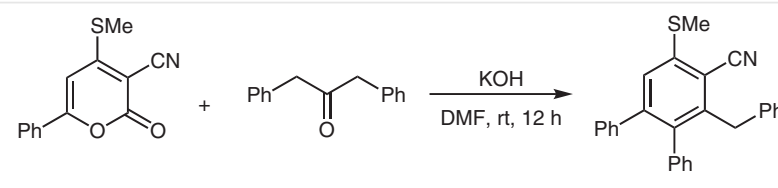

$5 a$

10

12a: $62 \%$

Scheme 2 The ring transformation of $2 \mathrm{H}$-pyran-2-ones 5 a with 1,3-diphenylacetone (10)

ing electron-donating functionalities (Table 5 , entries 6-8). Additionally, the reaction was found to be slightly slower when substrates $\mathbf{8 j}$ and $\mathbf{8 1}$ having methyl group at C- 5 position were used and reaction products were obtained in $74 \%$ and $79 \%$ yield, respectively (entries 10 and 11 ). All the synthesized compounds were characterized by spectroscopic analysis.

Finally, the reaction of substrate $\mathbf{5 a}$ was performed with 1,3-diphenylacetone (10) under similar reaction conditions. Expectedly, the reaction suffered from some undesired side reactions probably due to the presence of good leaving SMe at $\mathrm{C}-4$ position in substrate $\mathbf{5 a}$, which makes this position more vulnerable towards the nucleophile. The ring transformation product 12a was isolated in $62 \%$ yield (Scheme 2). Unfortunately, we could not isolate any side product from the reaction mixture. The isolated compound 12a was characterized as $3^{\prime}$-benzyl-5'-(methylthio)-[1,1':2',1"-terphenyl]-4'-carbonitrile by its spectroscopic analysis.

The possible mechanism for the ring transformation of $2 \mathrm{H}$-pyran-2-ones 8 with 4-phenylbutan-2-one (6) to diarylmethanes $\mathbf{9}$ is described in Scheme $3 .{ }^{27}$ Initially, the formation of bicyclic intermediate $\mathbf{1 3}$ takes place by the nucleophilic attack of anion generated from ketone 6 to the C-6 position of $2 \mathrm{H}$-pyran-2-ones $\mathbf{8}$, followed by intramolecular cyclization involving the carbonyl functionality of $\mathbf{6}$ and C-3 of the pyranone ring. Furthermore, the bicyclic intermediate $\mathbf{1 3}$ transforms to final product $\mathbf{9}$ on decarboxylation followed by dehydration.

In conclusion, we have developed a facile metal-free synthetic methodology for the synthesis of functionalized biaryl-cored diarylmethanes 9 through carbanion-induced ring transformation of 6-aryl-2H-pyran-2-ones $\mathbf{8}$ in good yields. In addition, the same approach was employed to achieve the synthesis of teraryl-cored diarylmethanes $\mathbf{1 1}$. Our methodology for the synthesis of functionalized diarylmethanes is simple, economical and does not require any toxic transition metal. Further investigations about this ring transformation approach are currently in progress.

Melting points were measured with REMI DDMS 2545 melting point apparatus. IR spectra were recorded with a Thermo Scientific Nicolet Nexus 470FT-IR spectrophotometer and band positions are reported in reciprocal centimeters. Samples were subjected to ATR mode to record the IR data. ${ }^{1} \mathrm{H}$ NMR and ${ }^{13} \mathrm{C}$ NMR spectra were recorded on a Bruker AV-400 spectrometer using the solvents indicated at 400 and 


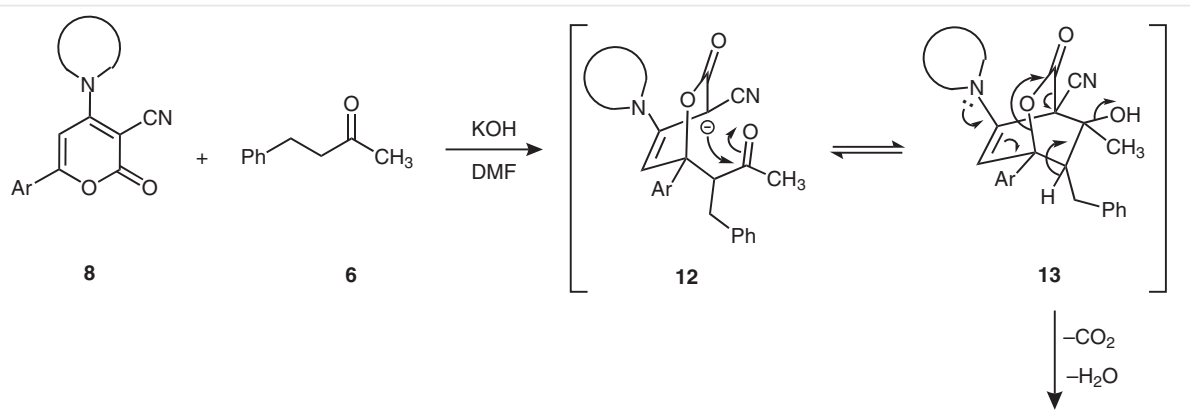<smiles>Cc1c(C#N)c(N2CCCCC2)cc(Br)c1Cc1ccccc1</smiles>

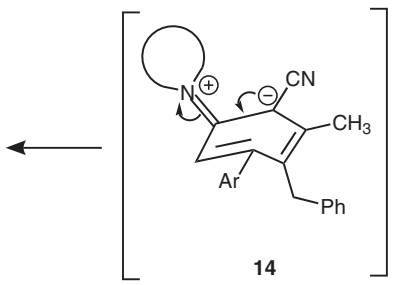

Scheme 3 Proposed mechanism for the synthesis of diarylmethanes 9 by the ring transformation of $2 \mathrm{H}$-pyran-2-ones 8 with 4-phenylbutan-2-one (6)

$100 \mathrm{MHz}$, respectively. Mass spectra $(\mathrm{m} / \mathrm{z})$ were recorded under the conditions of electron ionization (EI). All reactions were monitored by TLC that was performed on pre-coated sheets of silica gel 60 and column chromatography was performed with $\mathrm{Al}_{2} \mathrm{O}_{3}$ (neutral, 95\%) (Avra synthesis Pvt. Ltd.). Hexane and EtOAc were used as eluting solvents and bought from Avra Synthesis Pvt. Ltd. DMF was bought from Avra Synthesis Pvt. Ltd and was used without further purification.

\section{Diarylmethanes 7a-c; General Procedure}

A mixture of 3-cyano-4-methylsulfanyl-2H-pyran-2-one 5 (1.0 mmol, 1.0 equiv), 4-phenylbutan-2-one (6; $0.18 \mathrm{~mL}, 1.2 \mathrm{mmol}, 1.2$ equiv), and powdered $\mathrm{KOH}$ ( $84 \mathrm{mg}, 1.5 \mathrm{mmol}, 1.5$ equiv) in DMF ( $5 \mathrm{~mL}$ ) was stirred at r.t. for $10-12 \mathrm{~h}$. The course of reaction was monitored by TLC. On completion of the reaction, few ice pieces were added to the reaction mixture and neutralized with aq $2 \mathrm{M} \mathrm{HCl}$. The mixture was extracted with EtOAc $(3 \times 10 \mathrm{~mL})$, the combined organic layers were dried (anhyd $\mathrm{Na}_{2} \mathrm{SO}_{4}$ ), filtered, and concentrated under vacuum. The crude residue was purified by neutral alumina column chromatography using EtOAc-hexane (1:49) as an eluent and isolated products were characterized as diarylmethanes 7 by their spectroscopic analysis (Table 1).

\section{2-Benzyl-3-methyl-5-(methylthio)[1,1'-biphenyl]-4-carbonitrile}

(7a)

White solid; yield: $164 \mathrm{mg}(0.5 \mathrm{mmol}, 50 \%) ; \mathrm{mp} 148-150{ }^{\circ} \mathrm{C} ; R_{f}=0.5$ (EtOAc-hexane 1:49).

IR (ATR): $2212 \mathrm{~cm}^{-1}(\mathrm{C} \equiv \mathrm{N})$.

${ }^{1} \mathrm{H}$ NMR (400 MHz, CDCl $): \delta=2.33\left(\mathrm{~s}, 3 \mathrm{H}, \mathrm{CH}_{3}\right), 2.46\left(\mathrm{~s}, 3 \mathrm{H}, \mathrm{SCH}_{3}\right)$, $3.88\left(\mathrm{~s}, 2 \mathrm{H}, \mathrm{CH}_{2}\right), 6.81(\mathrm{~d}, J=7.2 \mathrm{~Hz}, 2 \mathrm{H}, \mathrm{ArH}), 6.98(\mathrm{~s}, 1 \mathrm{H}, \mathrm{ArH}), 7.05-$ $7.20(\mathrm{~m}, 5 \mathrm{H}, \mathrm{ArH}), 7.22-7.28(\mathrm{~m}, 3 \mathrm{H}, \mathrm{ArH})$.

${ }^{13} \mathrm{C}$ NMR $\left(100 \mathrm{MHz}, \mathrm{CDCl}_{3}\right): \delta=15.9,18.9,35.8,112.1,116.6,125.4$, 126.1, 127.7, 127.9, 128.3, 128.5, 128.6, 133.8, 139.6, 140.6, 141.2, 143.3, 147.8 .

GC-MS: $m / z=330[\mathrm{M}+1]^{+}$.
Anal. Calcd for $\mathrm{C}_{22} \mathrm{H}_{19} \mathrm{NS}$ : C, 80.20; H, 5.81; N, 4.25; S, 9.73. Found: C, 79.75; H, 5.83; N, 4.20; S, 9.06 .

2-Benzyl-4'-bromo-3-methyl-5-(methylthio)[1,1'-biphenyl]-4carbonitrile (7b)

White solid; yield: $212 \mathrm{mg}$ (0.52 mmol, 52\%); mp 150-152 ${ }^{\circ} \mathrm{C} ; R_{f}=0.5$ (EtOAc-hexane 1:49).

IR (ATR): $2214 \mathrm{~cm}^{-1}(\mathrm{C} \equiv \mathrm{N})$.

${ }^{1} \mathrm{H}$ NMR (400 MHz, $\left.\mathrm{CDCl}_{3}\right): \delta=2.34\left(\mathrm{~s}, 3 \mathrm{H}, \mathrm{CH}_{3}\right), 2.46\left(\mathrm{~s}, 3 \mathrm{H}, \mathrm{SCH}_{3}\right)$, $3.86\left(\mathrm{~s}, 2 \mathrm{H}, \mathrm{CH}_{2}\right), 6.79(\mathrm{~d}, J=6.8 \mathrm{~Hz}, 2 \mathrm{H}, \mathrm{ArH}), 6.94(\mathrm{~s}, 1 \mathrm{H}, \mathrm{ArH}), 6.97$ (td, $\left.J_{1}=8.8 \mathrm{~Hz}, J_{2}=2.0 \mathrm{~Hz}, 2 \mathrm{H}, \mathrm{ArH}\right), 7.07-7.20(\mathrm{~m}, 3 \mathrm{H}, \mathrm{ArH}), 7.38(\mathrm{td}$, $\left.J_{1}=8.8 \mathrm{~Hz}, J_{2}=2.0 \mathrm{~Hz}, 2 \mathrm{H}, \mathrm{ArH}\right)$.

${ }^{13} \mathrm{C}$ NMR $\left(100 \mathrm{MHz}, \mathrm{CDCl}_{3}\right): \delta=15.8,18.9,35.7,112.4,116.5,122.2$, $125.1,126.3,127.6,128.7,130.2,131.5,133.6,139.3,139.4,141.5$, 143.4, 146.5 .

GC-MS: $m / z=409[\mathrm{M}+1]^{+}, 410[\mathrm{M}+2]^{+}$.

Anal. Calcd for $\mathrm{C}_{22} \mathrm{H}_{18}$ BrNS: C, 64.71; H, 4.44; N, 3.43; S, 7.85. Found: C, 64.69; H, 4.50; N, 3.39; S, 7.81.

2-Benzyl-4'-methoxy-3-methyl-5-(methylthio)[1,1'-biphenyl]-4carbonitrile (7c)

White solid; yield: $204 \mathrm{mg}$ (0.57 mmol, 57\%); mp $144-146{ }^{\circ} \mathrm{C} ; R_{f}=0.5$ (EtOAc-hexane 1:49).

IR (ATR): $2214 \mathrm{~cm}^{-1}(\mathrm{C} \equiv \mathrm{N})$.

${ }^{1} \mathrm{H}$ NMR $\left(400 \mathrm{MHz}, \mathrm{CDCl}_{3}\right): \delta=2.32\left(\mathrm{~s}, 3 \mathrm{H}, \mathrm{CH}_{3}\right), 2.46\left(\mathrm{~s}, 3 \mathrm{H}, \mathrm{SCH}_{3}\right)$, $3.73\left(\mathrm{~s}, 3 \mathrm{H}, \mathrm{OCH}_{3}\right), 3.90\left(\mathrm{~s}, 2 \mathrm{H}, \mathrm{CH}_{2}\right), 6.78\left(\mathrm{td}, J_{1}=8.8 \mathrm{~Hz}, J_{2}=2.0 \mathrm{~Hz}, 2\right.$ $\mathrm{H}, \mathrm{ArH}), 6.82(\mathrm{~d}, J=7.2 \mathrm{~Hz}, 2 \mathrm{H}, \mathrm{ArH}), 6.98(\mathrm{~s}, 1 \mathrm{H}, \mathrm{ArH}), 7.03\left(\mathrm{td}, J_{1}=\right.$ $\left.8.8 \mathrm{~Hz}, J_{2}=2.0 \mathrm{~Hz}, 2 \mathrm{H}, \mathrm{ArH}\right), 7.08-7.20(\mathrm{~m}, 3 \mathrm{H}, \mathrm{ArH})$.

${ }^{13} \mathrm{C}$ NMR $\left(100 \mathrm{MHz}, \mathrm{CDCl}_{3}\right): \delta=15.9,18.9,35.8,55.3,111.8,113.7$, 116.7, 125.6, 126.1, 127.7, 128.6, 129.7, 132.9, 133.9, 139.7, 141.1, $143.3,147.5,159.3$

GC-MS: $m / z=360[\mathrm{M}+1]^{+}$.

Anal. Calcd for $\mathrm{C}_{23} \mathrm{H}_{21} \mathrm{NOS}$ : C, 76.85; H, 5.89; N, 3.90; S, 8.92. Found: C, 76.10; H, 5.84; N, 3.84; S, 8.58. 


\section{Biaryl-Cored Diarylmethanes 9a-k; General Procedure}

A mixture of 2H-pyran-2-one 8 (1.0 mmol, 1.0 equiv), 4-phenylbu$\tan$-2-one (6; $0.18 \mathrm{~mL}, 1.2 \mathrm{mmol}, 1.2$ equiv), and powdered $\mathrm{KOH}$ (84 $\mathrm{mg}, 1.5 \mathrm{mmol}, 1.5$ equiv) in DMF (5 mL) was stirred at r.t. for $10-14 \mathrm{~h}$. The course of reaction was monitored by TLC. On completion of the reaction, few ice pieces were added to the reaction mixture and neutralized with aq $2 \mathrm{M} \mathrm{HCl}$. The reaction mixture was extracted with EtOAc $(3 \times 10 \mathrm{~mL})$, the combined organic layers were dried (anhyd $\mathrm{Na}_{2} \mathrm{SO}_{4}$ ), filtered, and concentrated under vacuum. The crude residue was purified by neutral alumina column chromatography using EtOAc-hexane (1:49) as an eluent and isolated products were characterized as biaryl-cored diarylmethanes 9 by their spectroscopic analysis (Table 4).

\section{2-Benzyl-3-methyl-5-(piperidin-1-yl)[1,1'-biphenyl]-4-carboni- trile (9a)}

White solid; yield: $311 \mathrm{mg}, 0.85 \mathrm{mmol}$ (85\%); $\mathrm{mp} 114-116{ }^{\circ} \mathrm{C} ; R_{f}=0.5$ (EtOAc-hexane 1:49).

IR (ATR): $2215 \mathrm{~cm}^{-1}(\mathrm{C} \equiv \mathrm{N})$.

${ }^{1} \mathrm{H} \mathrm{NMR}\left(400 \mathrm{MHz}, \mathrm{CDCl}_{3}\right): \delta=1.46-1.54\left(\mathrm{~m}, 2 \mathrm{H}, \mathrm{CH}_{2}\right), 1.66-1.75(\mathrm{~m}$, $\left.4 \mathrm{H}, 2 \times \mathrm{CH}_{2}\right), 2.30\left(\mathrm{~s}, 3 \mathrm{H}, \mathrm{CH}_{3}\right), 3.06\left(\mathrm{t}, J=5.2 \mathrm{~Hz}, 4 \mathrm{H}, 2 \times \mathrm{NCH}_{2}\right), 3.84$ $\left(\mathrm{s}, 2 \mathrm{H}, \mathrm{CH}_{2}\right), 6.70(\mathrm{~s}, 1 \mathrm{H}, \mathrm{ArH}), 6.82(\mathrm{~d}, J=7.6 \mathrm{~Hz}, 2 \mathrm{H}, \mathrm{ArH}), 7.03-7.18$ (m, $5 \mathrm{H}, \mathrm{ArH}), 7.20-7.25(\mathrm{~m}, 3 \mathrm{H}, \mathrm{ArH})$.

${ }^{13} \mathrm{C}$ NMR $\left(100 \mathrm{MHz}, \mathrm{CDCl}_{3}\right): \delta=18.9,24.1,26.3,35.7,53.6,107.5$, $118.1,118.4,125.9,127.5,127.8,128.2,128.5,128.6,129.6,140.3$, $141.4,143.4,148.1,155.6$.

GC-MS: $m / z=367[\mathrm{M}+1]^{+}$.

Anal. Calcd for $\mathrm{C}_{26} \mathrm{H}_{26} \mathrm{~N}_{2}$ : C, 85.21; H, 7.15; N, 7.64. Found: C, 85.04; H, 7.18; N, 7.52.

\section{2-Benzyl-3-methyl-5-(4-phenylpiperazin-1-yl)[1,1'-biphenyl]-4- carbonitrile (9b)}

White solid; yield: $363 \mathrm{mg}$ (0.82 mmol, $82 \%) ; \mathrm{mp} 180-182{ }^{\circ} \mathrm{C} ; R_{f}=0.5$ (EtOAc-hexane 1:49).

IR (ATR): $2210 \mathrm{~cm}^{-1}(\mathrm{C} \equiv \mathrm{N})$.

${ }^{1} \mathrm{H} \mathrm{NMR}\left(400 \mathrm{MHz}, \mathrm{CDCl}_{3}\right): \delta=2.32\left(\mathrm{~s}, 3 \mathrm{H}, \mathrm{CH}_{3}\right), 3.25-3.35(\mathrm{~m}, 8 \mathrm{H}$, $\left.4 \times \mathrm{NCH}_{2}\right), 3.86\left(\mathrm{~s}, 2 \mathrm{H}, \mathrm{CH}_{2}\right), 6.76(\mathrm{~s}, 1 \mathrm{H}, \mathrm{ArH}), 6.81(\mathrm{t}, J=7.6 \mathrm{~Hz}, 3 \mathrm{H}$, ArH), 6.89 (d, J = 8.4 Hz, 2 H, ArH), 7.03-7.20 (m, 7 H, ArH), 7.21-7.26 (m, $3 \mathrm{H}, \mathrm{ArH})$.

${ }^{13} \mathrm{C}$ NMR $\left(100 \mathrm{MHz}, \mathrm{CDCl}_{3}\right): \delta=19.0,24.2,35.7,49.6,51.9,107.6$, $116.4,117.9,118.3,120.1,126.0,127.7,127.8,128.3,128.5,128.6$, 129.2, 130.6, 140.1, 141.2, 143.8, 148.4, 151.2, 154.1 .

GC-MS: $m / z=444[\mathrm{M}+1]^{+}$.

Anal. Calcd for $\mathrm{C}_{31} \mathrm{H}_{29} \mathrm{~N}_{3}$ : C, 83.94; $\mathrm{H}, 6.59 ; \mathrm{N}, 9.47$. Found: C, 83.55; $\mathrm{H}$, $6.58 ; \mathrm{N}, 9.37$.

\section{2-Benzyl-4'-chloro-3-methyl-5-(piperidin-1-yl)[1,1'-biphenyl]-4- carbonitrile (9c)}

White solid; yield: $312 \mathrm{mg}$ (0.77 mmol, 78\%); mp 141-143 ${ }^{\circ} \mathrm{C} ; R_{f}=0.5$ (EtOAc-hexane 1:49).

IR (ATR): $2218 \mathrm{~cm}^{-1}(\mathrm{C} \equiv \mathrm{N})$.

${ }^{1} \mathrm{H}$ NMR $\left(400 \mathrm{MHz}, \mathrm{CDCl}_{3}\right): \delta=1.46-1.57\left(\mathrm{~m}, 2 \mathrm{H}, \mathrm{CH}_{2}\right), 1.66-1.75(\mathrm{~m}$, $\left.4 \mathrm{H}, 2 \times \mathrm{CH}_{2}\right), 2.30\left(\mathrm{~s}, 3 \mathrm{H}, \mathrm{CH}_{3}\right), 3.06\left(\mathrm{t}, J=5.6 \mathrm{~Hz}, 4 \mathrm{H}, 2 \times \mathrm{NCH}_{2}\right), 3.81$ $\left(\mathrm{s}, 2 \mathrm{H}, \mathrm{CH}_{2}\right), 6.65$ (s, $\left.1 \mathrm{H}, \mathrm{ArH}\right), 6.80(\mathrm{~d}, J=7.2 \mathrm{~Hz}, 2 \mathrm{H}, \mathrm{ArH}), 7.01$ (d, J = $8.4 \mathrm{~Hz}, 2 \mathrm{H}, \mathrm{ArH}), 7.04-7.21$ (m, $5 \mathrm{H}, \mathrm{ArH})$.
${ }^{13} \mathrm{C}$ NMR $\left(100 \mathrm{MHz}, \mathrm{CDCl}_{3}\right): \delta=18.9,24.1,26.2,35.6,53.5,107.7$, 117.9, 118.2, 126.0, 127.7, 128.4, 128.6, 129.4, 129.9, 133.7, 139.8, 140.0, 143.5, 146.9, 155.7.

GC-MS: $m / z=401[\mathrm{M}+1]^{+}, 402[\mathrm{M}+2]^{+}$.

Anal. Calcd for $\mathrm{C}_{26} \mathrm{H}_{25} \mathrm{ClN}_{2}$ : C, 77.89; H, 6.28; N, 6.99. Found: C, 77.39; $\mathrm{H}, 6.41 ; \mathrm{N}, 6.43$.

\section{2-Benzyl-4'-bromo-3-methyl-5-(piperidin-1-yl)[1,1'-biphenyl]-4- carbonitrile (9d)}

White solid; yield: $364 \mathrm{mg}$ (0.82 mmol, 82\%); $\mathrm{mp} 116-118^{\circ} \mathrm{C} ; R_{f}=0.5$ (EtOAc-hexane 1:49).

IR (ATR): $2217 \mathrm{~cm}^{-1}(\mathrm{C} \equiv \mathrm{N})$.

${ }^{1} \mathrm{H}$ NMR $\left(400 \mathrm{MHz}, \mathrm{CDCl}_{3}\right): \delta=1.47-1.56\left(\mathrm{~m}, 2 \mathrm{H}, \mathrm{CH}_{2}\right), 1.66-1.73(\mathrm{~m}$, $\left.4 \mathrm{H}, 2 \times \mathrm{CH}_{2}\right), 2.30\left(\mathrm{~s}, 3 \mathrm{H}, \mathrm{CH}_{3}\right), 3.06\left(\mathrm{t}, J=4.8 \mathrm{~Hz}, 4 \mathrm{H}, 2 \times \mathrm{NCH}_{2}\right), 3.81$ $\left(\mathrm{s}, 2 \mathrm{H}, \mathrm{CH}_{2}\right), 6.65(\mathrm{~s}, 1 \mathrm{H}, \mathrm{ArH}), 6.80(\mathrm{~d}, J=7.6 \mathrm{~Hz}, 2 \mathrm{H}, \mathrm{ArH}), 6.95(\mathrm{~d}, J=$ $8.0 \mathrm{~Hz}, 2 \mathrm{H}, \mathrm{ArH}), 7.03-7.19(\mathrm{~m}, 3 \mathrm{H}, \mathrm{ArH}), 7.34$ (d, J = 8.0 Hz, $2 \mathrm{H}$, $\mathrm{ArH})$.

${ }^{13} \mathrm{C}$ NMR $\left(100 \mathrm{MHz}, \mathrm{CDCl}_{3}\right): \delta=18.9,24.1,26.2,35.6,53.5,107.7$, $117.9,118.1,121.9,126.1,127.7,128.6,129.4,130.2,131.3,140.0$, 140.2, 143.6, 146.8, 155.7.

GC-MS: $m / z=446[\mathrm{M}+1]^{+}, 447[\mathrm{M}+2]^{+}$.

Anal. Calcd for $\mathrm{C}_{26} \mathrm{H}_{25} \mathrm{BrN}_{2}$ : C, 70.11; $\mathrm{H}, 5.66 ; \mathrm{N}, 6.29$. Found: C, 70.03; H, 5.66; N, 6.11.

\section{2-Benzyl-4'-bromo-3-methyl-5-(4-phenylpiperazin-1-yl)[1,1'-bi- phenyl]-4-carbonitrile (9e)}

White solid; yield: $417 \mathrm{mg}(0.79 \mathrm{mmol}, 80 \%) ; \mathrm{mp} 172-174{ }^{\circ} \mathrm{C} ; R_{f}=0.5$ (EtOAc-hexane 1:49).

IR (ATR): $2210 \mathrm{~cm}^{-1}(\mathrm{C} \equiv \mathrm{N})$.

${ }^{1} \mathrm{H}$ NMR $\left(400 \mathrm{MHz}, \mathrm{CDCl}_{3}\right): \delta=2.34\left(\mathrm{~s}, 3 \mathrm{H}, \mathrm{CH}_{3}\right), 3.25-3.36(\mathrm{~m}, 8 \mathrm{H}$, $\left.4 \times \mathrm{NCH}_{2}\right), 3.84\left(\mathrm{~s}, 2 \mathrm{H}, \mathrm{CH}_{2}\right), 6.71(\mathrm{~s}, 1 \mathrm{H}, \mathrm{ArH}), 6.79-6.85(\mathrm{~m}, 3 \mathrm{H}$, $\operatorname{ArH}), 6.91(\mathrm{~d}, J=8.0 \mathrm{~Hz}, 2 \mathrm{H}, \mathrm{ArH}), 6.97\left(\mathrm{td}, J_{1}=8.4 \mathrm{~Hz}, J_{2}=1.6 \mathrm{~Hz}, 2 \mathrm{H}\right.$, ArH), 7.06-7.25 (m, $5 \mathrm{H}, \mathrm{ArH}), 7.37\left(\mathrm{td}, J_{1}=8.4 \mathrm{~Hz}, J_{2}=1.6 \mathrm{~Hz}, 2 \mathrm{H}\right.$, $\mathrm{ArH})$.

${ }^{13} \mathrm{C}$ NMR $\left(100 \mathrm{MHz}, \mathrm{CDCl}_{3}\right): \delta=18.9,35.6,49.6,51.9,107.8,116.4$, 117.7, 118.1, 120.1, 122.0, 126.1, 127.7, 128.6, 129.2, 130.2, 130.4, 131.4, 139.8, 140.0, 143.9, 147.1, 151.2, 154.2.

GC-MS: $m / z=523[\mathrm{M}+1]^{+}, 524[\mathrm{M}+2]^{+}$.

Anal. Calcd for $\mathrm{C}_{31} \mathrm{H}_{28} \mathrm{BrN}_{3}$ : C, 71.26; $\mathrm{H}, 5.40 ; \mathrm{N}, 8.04$. Found: C, 71.19; H, 5.47; N, 7.91.

\section{2-Benzyl-3,4'-dimethyl-5-(piperidin-1-yl)[1,1'-biphenyl]-4-carbo- nitrile (9f)}

White solid; yield: $357 \mathrm{mg}$ (0.94 mmol, 94\%); $\mathrm{mp} 162-164^{\circ} \mathrm{C} ; R_{f}=0.5$ (EtOAc-hexane 1:49).

IR (ATR): $2215 \mathrm{~cm}^{-1}(\mathrm{C} \equiv \mathrm{N})$.

${ }^{1} \mathrm{H}$ NMR $\left(400 \mathrm{MHz}, \mathrm{CDCl}_{3}\right): \delta=1.45-1.56\left(\mathrm{~m}, 2 \mathrm{H}, \mathrm{CH}_{2}\right), 1.64-1.75(\mathrm{~m}$, $\left.4 \mathrm{H}, 2 \times \mathrm{CH}_{2}\right), 2.26\left(\mathrm{~s}, 3 \mathrm{H}, \mathrm{CH}_{3}\right), 2.28\left(\mathrm{~s}, 3 \mathrm{H}, \mathrm{CH}_{3}\right), 3.05$ (t, $J=5.2 \mathrm{~Hz}, 4$ $\left.\mathrm{H}, 2 \times \mathrm{NCH}_{2}\right), 3.85\left(\mathrm{~s}, 2 \mathrm{H}, \mathrm{CH}_{2}\right), 6.70(\mathrm{~s}, 1 \mathrm{H}, \mathrm{ArH}), 6.83(\mathrm{~d}, J=7.6 \mathrm{~Hz}, 2$ $\mathrm{H}, \mathrm{ArH}), 6.95-7.10$ (m, $5 \mathrm{H}, \mathrm{ArH}), 7.14$ (t, J = 7.6 Hz, $2 \mathrm{H}, \mathrm{ArH})$.

${ }^{13} \mathrm{C}$ NMR $\left(100 \mathrm{MHz}, \mathrm{CDCl}_{3}\right): \delta=18.9,21.2,24.2,26.3,35.7,53.6,107.3$, $118.1,118.4,125.9,127.8,128.5,128.9,129.7,137.3,138.5,140.4$, 143.4, 148.1, 148.2, 155.6 .

GC-MS: $m / z=381[\mathrm{M}+1]^{+}$.

Anal. Calcd for $\mathrm{C}_{27} \mathrm{H}_{28} \mathrm{~N}_{2}$ : C, 85.22; H, 7.42; N, 7.36. Found: C, 84.71; H, 7.42; N, 7.16. 
2-Benzyl-4'-methoxy-3-methyl-5-(piperidin-1-yl)[1,1'-biphenyl]4-carbonitrile (9g)

White solid; yield: $356 \mathrm{mg}$ (0.90 mmol, 90\%); $\mathrm{mp} 172-174{ }^{\circ} \mathrm{C} ; R_{f}=0.5$ (EtOAc-hexane 1:49).

IR (ATR): $2213 \mathrm{~cm}^{-1}(\mathrm{C} \equiv \mathrm{N})$.

${ }^{1} \mathrm{H}$ NMR $\left(400 \mathrm{MHz}, \mathrm{CDCl}_{3}\right): \delta=1.45-1.58\left(\mathrm{~m}, 2 \mathrm{H}, \mathrm{CH}_{2}\right), 1.66-1.75(\mathrm{~m}$, $\left.4 \mathrm{H}, 2 \times \mathrm{CH}_{2}\right), 2.28\left(\mathrm{~s}, 3 \mathrm{H}, \mathrm{CH}_{3}\right), 3.05\left(\mathrm{t}, J=5.2 \mathrm{~Hz}, 4 \mathrm{H}, 2 \times \mathrm{NCH}_{2}\right), 3.71$ (s, $\left.3 \mathrm{H}, \mathrm{OCH}_{3}\right), 3.86\left(\mathrm{~s}, 2 \mathrm{H}, \mathrm{CH}_{2}\right), 6.70(\mathrm{~s}, 1 \mathrm{H}, \mathrm{ArH}), 6.75(\mathrm{~d}, J=8.0 \mathrm{~Hz}, 2$ $\mathrm{H}, \operatorname{ArH}), 6.83(\mathrm{~d}, J=7.2 \mathrm{~Hz}, 2 \mathrm{H}, \operatorname{ArH}), 7.02(\mathrm{~d}, J=8.0 \mathrm{~Hz}, 2 \mathrm{H}, \mathrm{ArH})$, 7.04-7.10 (m, $1 \mathrm{H}, \mathrm{ArH}), 7.14$ (t, J= 7.2 Hz, $2 \mathrm{H}, \mathrm{ArH})$.

${ }^{13} \mathrm{C}$ NMR $\left(100 \mathrm{MHz}, \mathrm{CDCl}_{3}\right): \delta=18.9,24.2,26.3,35.7,53.6,55.3,107.2$, 113.6, 118.2, 118.5, 125.9, 127.8, 128.5, 129.7, 133.8, 140.4, 143.3, 147.9, 155.6, 159.1 .

GC-MS: $m / z=397[\mathrm{M}+1]^{+}$.

Anal. Calcd for $\mathrm{C}_{27} \mathrm{H}_{28} \mathrm{~N}_{2} \mathrm{O}$ : C, 81.78; H, 7.12; N, 7.06. Found: C, 81.11; H, 7.14; N, 6.92.

\section{2-Benzyl-4'-methoxy-3-methyl-5-(4-phenylpiperazin-1-yl)[1,1'- biphenyl]-4-carbonitrile (9h)}

White solid; yield: $435 \mathrm{mg}$ (0.92 mmol, $92 \%) ; \mathrm{mp} 174-176{ }^{\circ} \mathrm{C} ; R_{f}=0.5$ (EtOAc-hexane 1:49).

IR (ATR): $2214 \mathrm{~cm}^{-1}(\mathrm{C} \equiv \mathrm{N})$.

${ }^{1} \mathrm{H}$ NMR (400 MHz, $\left.\mathrm{CDCl}_{3}\right): \delta=2.31\left(\mathrm{~s}, 3 \mathrm{H}, \mathrm{CH}_{3}\right), 3.25-3.36(\mathrm{~m}, 8 \mathrm{H}$, $\left.4 \times \mathrm{NCH}_{2}\right), 3.72\left(\mathrm{~s}, 3 \mathrm{H}, \mathrm{OCH}_{3}\right), 3.89\left(\mathrm{~s}, 2 \mathrm{H}, \mathrm{CH}_{2}\right), 6.75-6.77(\mathrm{~m}, 2 \mathrm{H}$, $\operatorname{ArH}), 6.78(\mathrm{~s}, 1 \mathrm{H}, \mathrm{ArH}), 6.81-6.87(\mathrm{~m}, 2 \mathrm{H}, \mathrm{ArH}), 6.88-6.95(\mathrm{~m}, 2 \mathrm{H}$, $\operatorname{ArH}), 7.04(\mathrm{~d}, J=8.8 \mathrm{~Hz}, 2 \mathrm{H}, \mathrm{ArH}), 7.06-7.12(\mathrm{~m}, 1 \mathrm{H}, \mathrm{ArH}), 7.13-7.24$ (m, $5 \mathrm{H}, \mathrm{ArH})$.

${ }^{13} \mathrm{C}$ NMR $\left(100 \mathrm{MHz}, \mathrm{CDCl}_{3}\right): \delta=19.0,35.7,49.6,51.9,55.3,107.3$, 113.7, 116.4, 118.5, 120.1, 126.0, 127.8, 128.5, 128.7, 129.2, 129.7, $130.8,133.5,140.2,143.7,148.1,151.2,154.1,159.2$.

GC-MS: $m / z=474[\mathrm{M}+1]^{+}$.

Anal. Calcd for $\mathrm{C}_{32} \mathrm{H}_{31} \mathrm{~N}_{3} \mathrm{O}$ : C, 81.15; H, 6.60; N, 8.87. Found: C, 81.02; $\mathrm{H}, 7.12 ; \mathrm{N}, 8.71$.

\section{3-Benzyl-2-methyl-4-(naphthalen-2-yl)-6-(piperidin-1-yl)benzo- nitrile (9i)}

White solid; yield: $366 \mathrm{mg}$ (0.88 mmol, 88\%); $\mathrm{mp} 162-164{ }^{\circ} \mathrm{C} ; R_{f}=0.5$ (EtOAc-hexane 1:49).

IR (ATR): $2213 \mathrm{~cm}^{-1}(\mathrm{C} \equiv \mathrm{N})$.

${ }^{1} \mathrm{H} \mathrm{NMR}\left(400 \mathrm{MHz}, \mathrm{CDCl}_{3}\right): \delta=1.44-1.55\left(\mathrm{~m}, 2 \mathrm{H}, \mathrm{CH}_{2}\right), 1.66-1.76(\mathrm{~m}$, $\left.4 \mathrm{H}, 2 \times \mathrm{CH}_{2}\right), 2.33\left(\mathrm{~s}, 3 \mathrm{H}, \mathrm{CH}_{3}\right), 3.07\left(\mathrm{t}, J=5.2 \mathrm{~Hz}, 4 \mathrm{H}, 2 \times \mathrm{NCH}_{2}\right), 3.87$ $\left(\mathrm{s}, 2 \mathrm{H}, \mathrm{CH}_{2}\right), 6.79(\mathrm{~s}, 1 \mathrm{H}, \mathrm{ArH}), 6.82(\mathrm{~d}, J=6.8 \mathrm{~Hz}, 2 \mathrm{H}, \mathrm{ArH}), 7.07(\mathrm{~d}, J=$ $6.8 \mathrm{~Hz}, 1 \mathrm{H}, \mathrm{ArH}), 7.13(\mathrm{t}, J=7.6 \mathrm{~Hz}, 2 \mathrm{H}, \mathrm{ArH}), 7.21\left(\mathrm{dd}, J_{1}=8.4 \mathrm{~Hz}, J_{2}=\right.$ $1.6 \mathrm{~Hz}, 1 \mathrm{H}, \mathrm{ArH}), 7.35-7.41$ (m, $2 \mathrm{H}, \mathrm{ArH}), 7.54$ (s, $1 \mathrm{H}, \mathrm{ArH}), 7.58-$ $7.65(\mathrm{~m}, 1 \mathrm{H}, \mathrm{ArH}), 7.68(\mathrm{~d}, J=8.8 \mathrm{~Hz}, 1 \mathrm{H}, \mathrm{ArH}), 7.70-7.78(\mathrm{~m}, 1 \mathrm{H}$, $\mathrm{ArH})$.

${ }^{13} \mathrm{C}$ NMR $\left(100 \mathrm{MHz}, \mathrm{CDCl}_{3}\right): \delta=19.0,24.2,26.3,35.8,53.6,107.6$, 118.1, 118.6, 125.9, 126.3, 126.4, 126.8, 127.5, 127.6, 127.7, 127.8, $128.1,128.5,129.8,132.5,133.0,138.9,140.3,143.5,148.1,155.7$.

GC-MS: $m / z=417[\mathrm{M}+1]^{+}$.

Anal. Calcd for $\mathrm{C}_{30} \mathrm{H}_{28} \mathrm{~N}_{2}$ : C, 86.50; H, 6.78; N, 6.72. Found: $\mathrm{C}, 86.17 ; \mathrm{H}$, $6.77 ; \mathrm{N}, 6.60$.

\section{2-Benzyl-3,6-dimethyl-5-(4-phenylpiperazin-1-yl)[1,1'-biphenyl]-} 4-carbonitrile (9j)

White solid; yield: $356 \mathrm{mg}$ (0.78 mmol, 78\%); mp 140-142 ${ }^{\circ} \mathrm{C} ; R_{f}=0.5$ (EtOAc-hexane 1:49).
IR (ATR): $2215 \mathrm{~cm}^{-1}(\mathrm{C} \equiv \mathrm{N})$.

${ }^{1} \mathrm{H}$ NMR (400 MHz, $\left.\mathrm{CDCl}_{3}\right): \delta=1.89\left(\mathrm{~s}, 3 \mathrm{H}, \mathrm{CH}_{3}\right), 2.30\left(\mathrm{~s}, 3 \mathrm{H}, \mathrm{CH}_{3}\right)$, 3.18-3.55 (m, $\left.8 \mathrm{H}, 4 \times \mathrm{NCH}_{2}\right), 3.69\left(\mathrm{~s}, 2 \mathrm{H}, \mathrm{CH}_{2}\right), 6.72(\mathrm{~d}, J=7.2 \mathrm{~Hz}, 2 \mathrm{H}$, ArH), $6.79(\mathrm{t}, J=7.2 \mathrm{~Hz}, 1 \mathrm{H}, \mathrm{ArH}), 6.85-6.94(\mathrm{~m}, 4 \mathrm{H}, \mathrm{ArH}), 7.01-7.15$ (m, $3 \mathrm{H}, \mathrm{ArH}), 7.16-7.24(\mathrm{~m}, 5 \mathrm{H}, \mathrm{ArH})$.

${ }^{13} \mathrm{CNMR}\left(100 \mathrm{MHz}, \mathrm{CDCl}_{3}\right): \delta=16.9,18.5,36.6,50.5,50.6,111.7$, $116.6,118.4,119.9,125.8,127.3,127.8,128.2,128.3,128.5,129.1$, $133.5,134.3,139.8,140.1,140.2,148.9,151.5,151.8$.

GC-MS: $m / z=458[\mathrm{M}+1]^{+}$.

Anal. Calcd for $\mathrm{C}_{32} \mathrm{H}_{31} \mathrm{~N}_{3}$ : C, 83.99; H, 6.83; N, 9.18. Found: C, 83.67; $\mathrm{H}$, $6.87 ; \mathrm{N}, 8.99$.

\section{3-Benzyl-2-methyl-6-(piperidin-1-yl)-4-(thiophen-2-yl)benzoni- trile (9k)}

White solid; yield: $308 \mathrm{mg}$ (0.83 mmol (83\%); $\mathrm{mp} 115-117^{\circ} \mathrm{C} ; R_{f}=0.5$ (EtOAc-hexane 1:49).

IR (ATR): $2215 \mathrm{~cm}^{-1}(\mathrm{C} \equiv \mathrm{N})$.

${ }^{1} \mathrm{H}$ NMR $\left(400 \mathrm{MHz}, \mathrm{CDCl}_{3}\right): \delta=1.49-1.58\left(\mathrm{~m}, 2 \mathrm{H}, \mathrm{CH}_{2}\right), 1.67-1.76(\mathrm{~m}$, $\left.4 \mathrm{H}, 2 \times \mathrm{CH}_{2}\right), 2.30\left(\mathrm{~s}, 3 \mathrm{H}, \mathrm{CH}_{3}\right), 3.07\left(\mathrm{t}, J=5.2 \mathrm{~Hz}, 4 \mathrm{H}, 2 \times \mathrm{NCH}_{2}\right), 4.00$ $\left(\mathrm{s}, 2 \mathrm{H}, \mathrm{CH}_{2}\right), 6.78(\mathrm{ds}, J=3.2 \mathrm{~Hz}, 1 \mathrm{H}, \mathrm{ArH}), 6.85-6.91(\mathrm{~m}, 4 \mathrm{H}, \mathrm{ArH})$, $7.11(\mathrm{t}, J=7.2 \mathrm{~Hz}, 1 \mathrm{H}, \mathrm{ArH}), 7.14-7.25(\mathrm{~m}, 3 \mathrm{H}, \mathrm{ArH})$.

${ }^{13} \mathrm{C}$ NMR $\left(100 \mathrm{MHz}, \mathrm{CDCl}_{3}\right): \delta=19.0,24.1,26.2,35.9,53.5,107.9$, $117.9,119.2,126.0,126.1,126.9,127.3,127.8,128.6,130.2,140.2$, 140.4, 142.1, 143.7, 155.5.

GC-MS: $m / z=373[\mathrm{M}+1]^{+}$.

Anal. Calcd for $\mathrm{C}_{24} \mathrm{H}_{24} \mathrm{~N}_{2} \mathrm{~S}$ : C, 77.38; H, 6.49; N, 7.52; S, 8.61. Found: C, 77.68; H, 6.44; N, 7.43; S, 8.50.

\section{Teraryl-Cored Diarylmethanes 11a-j and 111; General Procedure}

A mixture of $2 \mathrm{H}$-pyran-2-one $\mathbf{8}$ (1.0 mmol, 1.0 equiv), 1,3-diphenylacetone (10; $0.24 \mathrm{~mL}, 1.2 \mathrm{mmol}, 1.2$ equiv), and powdered $\mathrm{KOH}(84 \mathrm{mg}$, $1.5 \mathrm{mmol}, 1.5$ equiv) in DMF ( $5 \mathrm{~mL}$ ) was stirred at r.t. for $10-15 \mathrm{~h}$. The progress of the reaction was checked by TLC. On completion of the reaction, few ice pieces were added to the reaction mixture and neutralized with aq $2 \mathrm{M} \mathrm{HCl}$. The reaction mixture was extracted with EtOAc $(3 \times 10 \mathrm{~mL})$, the combined organic layers were dried (anhyd $\mathrm{Na}_{2} \mathrm{SO}_{4}$ ), filtered, and evaporated under vacuum. The crude residue was purified by neutral alumina column chromatography using EtOAc-hexane (1:49) as an eluent and compounds were characterized as teraryl-cored diarylmethanes $\mathbf{1 1}$ by their spectroscopic analysis (Table 5).

\section{3'-Benzyl-5'-(piperidin-1-yl)[1,1':2',1'-terphenyl]-4'-carbonitrile} (11a)

White solid; yield: $376 \mathrm{mg}(0.88 \mathrm{mmol}, 88 \%) ; \mathrm{mp} 145-148{ }^{\circ} \mathrm{C} ; R_{f}=0.5$ (EtOAc-hexane 1:49).

IR (ATR): $2214 \mathrm{~cm}^{-1}(\mathrm{C} \equiv \mathrm{N})$.

${ }^{1} \mathrm{H}$ NMR $\left(400 \mathrm{MHz}, \mathrm{CDCl}_{3}\right): \delta=1.49-1.58\left(\mathrm{~m}, 2 \mathrm{H}, \mathrm{CH}_{2}\right), 1.69-1.78(\mathrm{~m}$, $\left.4 \mathrm{H}, 2 \times \mathrm{CH}_{2}\right), 3.13\left(\mathrm{t}, J=5.2 \mathrm{~Hz}, 4 \mathrm{H}, 2 \times \mathrm{NCH}_{2}\right), 4.06\left(\mathrm{~s}, 2 \mathrm{H}, \mathrm{CH}_{2}\right), 6.71-$ $6.81(\mathrm{~m}, 3 \mathrm{H}, \mathrm{ArH}), 6.86(\mathrm{~s}, 1 \mathrm{H}, \mathrm{ArH}), 6.90-6.95(\mathrm{~m}, 2 \mathrm{H}, \mathrm{ArH}), 6.96-$ 7.10 ( $\mathrm{m}, 9 \mathrm{H}, \mathrm{ArH}), 7.17-7.26$ (m, $1 \mathrm{H}, \mathrm{ArH})$.

${ }^{13} \mathrm{C}$ NMR $\left(100 \mathrm{MHz}, \mathrm{CDCl}_{3}\right): \delta=24.1,26.2,38.4,53.6,107.2,118.9$, 125.9, 126.8, 126.9, 127.6, 127.7, 128.1, 128.5, 129.4, 130.9, 135.0, $138.1,138.2,139.5,141.1,144.3,147.1,156.8$.

GC-MS: $m / z=429[\mathrm{M}+1]^{+}$.

Anal. Calcd for $\mathrm{C}_{31} \mathrm{H}_{28} \mathrm{~N}_{2}$ : C, 86.88; H, 6.59; N, 6.54. Found: C, 86.82; H, $6.69 ; \mathrm{N}, 5.97$. 
3'-Benzyl-5'-(4-phenylpiperazin-1-yl)[1,1':2',1"-terphenyl]-4'-carbonitrile (11b)

White solid; yield: $414 \mathrm{mg}$ (0.82 mmol, $82 \%) ; \mathrm{mp} 167-169{ }^{\circ} \mathrm{C} ; R_{f}=0.5$ (EtOAc-hexane 1:49).

IR (ATR): $2215 \mathrm{~cm}^{-1}(\mathrm{C} \equiv \mathrm{N})$.

${ }^{1} \mathrm{H}$ NMR $\left(400 \mathrm{MHz}, \mathrm{CDCl}_{3}\right): \delta=3.30-3.38\left(\mathrm{~m}, 8 \mathrm{H}, 4 \times \mathrm{NCH}_{2}\right), 4.08(\mathrm{~s}, 2$ $\left.\mathrm{H}, \mathrm{CH}_{2}\right), 6.72-6.84(\mathrm{~m}, 5 \mathrm{H}, \mathrm{ArH}), 6.88-6.96(\mathrm{~m}, 5 \mathrm{H}, \mathrm{ArH}), 6.99-7.07$ ( $\mathrm{m}, 9 \mathrm{H}, \mathrm{ArH}), 7.20(\mathrm{t}, J=7.6 \mathrm{~Hz}, 2 \mathrm{H}, \mathrm{ArH})$.

${ }^{13} \mathrm{C}$ NMR $\left(100 \mathrm{MHz}, \mathrm{CDCl}_{3}\right): \delta=38.5,49.6,52.0,107.3,116.5,117.9$, $118.9,120.1,126.1,126.9,127.1,127.7,127.8,128.2,128.5,129.2$, $129.4,130.8,135.9,137.9,139.3,140.8,144.7,147.4,151.2,155.3$.

GC-MS: $m / z=506[\mathrm{M}+1]^{+}$.

Anal. Calcd for $\mathrm{C}_{36} \mathrm{H}_{31} \mathrm{~N}_{3}: \mathrm{C}, 85.51 ; \mathrm{H}, 6.18 ; \mathrm{N}$, 8.31. Found: $\mathrm{C}, 85.02 ; \mathrm{H}$, $6.24 ; \mathrm{N}, 8.13$.

3'-Benzyl-4-chloro-5'-(piperidin-1-yl)[1,1':2',1"-terphenyl]-4'-carbonitrile (11c)

White solid; yield: $365 \mathrm{mg}$ (0.79 mmol, 79\%); mp 158-160 ${ }^{\circ} \mathrm{C} ; R_{f}=0.5$ (EtOAc-hexane 1:49).

IR (ATR): $2215 \mathrm{~cm}^{-1}(\mathrm{C} \equiv \mathrm{N})$.

${ }^{1} \mathrm{H} \mathrm{NMR}\left(400 \mathrm{MHz}, \mathrm{CDCl}_{3}\right): \delta=1.49-1.57\left(\mathrm{~m}, 2 \mathrm{H}, \mathrm{CH}_{2}\right), 1.67-1.78(\mathrm{~m}$, $\left.4 \mathrm{H}, 2 \times \mathrm{CH}_{2}\right), 3.12\left(\mathrm{t}, J=5.2 \mathrm{~Hz}, 4 \mathrm{H}, 2 \times \mathrm{NCH}_{2}\right), 4.04\left(\mathrm{~s}, 2 \mathrm{H}, \mathrm{CH}_{2}\right), 6.70-$ $6.78(\mathrm{~m}, 4 \mathrm{H}, \mathrm{ArH}), 6.81(\mathrm{~s}, 1 \mathrm{H}, \mathrm{ArH}), 6.85(\mathrm{~d}, J=8.4 \mathrm{~Hz}, 2 \mathrm{H}, \mathrm{ArH})$, 6.97-7.10 (m, $8 \mathrm{H}, \mathrm{ArH})$.

${ }^{13} \mathrm{C}$ NMR $\left(100 \mathrm{MHz}, \mathrm{CDCl}_{3}\right): \delta=24.1,26.2,38.4,53.5,107.4,118.0$, 118.7, 126.0, 127.0, 127.9, 128.1, 128.5, 128.7, 129.5, 130.7, 130.8, $134.9,137.9,139.3,139.5,144.6,145.8,156.8$.

GC-MS: $m / z=464[\mathrm{M}+1]^{+}, 465[\mathrm{M}+2]^{+}$.

Anal. Calcd for $\mathrm{C}_{31} \mathrm{H}_{27} \mathrm{ClN}_{2}$ : C, 80.42; $\mathrm{H}, 5.88 ; \mathrm{N}, 6.05$. Found: C, 80.59; H, 5.96; N, 5.48 .

3'-Benzyl-4-bromo-5'-(piperidin-1-yl)[1,1':2',1"-terphenyl]-4'-carbonitrile (11d)

White solid; yield: $415 \mathrm{mg}$ (0.82 mmol, $82 \%) ; \mathrm{mp} 150-152{ }^{\circ} \mathrm{C} ; R_{f}=0.5$ (EtOAc-hexane 1:49).

IR (ATR): $2217 \mathrm{~cm}^{-1}(\mathrm{C} \equiv \mathrm{N})$.

${ }^{1} \mathrm{H} \mathrm{NMR}\left(400 \mathrm{MHz}, \mathrm{CDCl}_{3}\right): \delta=1.49-1.57\left(\mathrm{~m}, 2 \mathrm{H}, \mathrm{CH}_{2}\right), 1.68-1.77(\mathrm{~m}$, $\left.4 \mathrm{H}, 2 \times \mathrm{CH}_{2}\right), 3.12\left(\mathrm{t}, J=5.2 \mathrm{~Hz}, 4 \mathrm{H}, 2 \times \mathrm{NCH}_{2}\right), 4.04\left(\mathrm{~s}, 2 \mathrm{H}, \mathrm{CH}_{2}\right), 6.69-$ 6.79 (m, 6 H, ArH), 6.80 (s, $1 \mathrm{H}, \mathrm{ArH}), 6.97-7.08$ (m, $6 \mathrm{H}, \mathrm{ArH}), 7.16$ (d, $J=8.4 \mathrm{~Hz}, 2 \mathrm{H}, \mathrm{ArH})$.

${ }^{13} \mathrm{C}$ NMR $\left(100 \mathrm{MHz}, \mathrm{CDCl}_{3}\right): \delta=24.1,26.2,38.4,53.5,107.5,118.0$, 118.7, 121.3, 126.0, 127.0, 127.9, 128.1, 128.5, 130.8, 131.0, 134.8, 137.9, 138.0, 139.3, 140.0, 144.6, 145.7, 156.8 .

GC-MS: $m / z=508[\mathrm{M}+1]^{+}, 509[\mathrm{M}+2]^{+}$.

Anal. Calcd for $\mathrm{C}_{31} \mathrm{H}_{27} \mathrm{BrN}_{2}$ : C, 73.37; H, 5.36; N, 5.52. Found: C, 73.53; H, 5.44; N, 5.35 .

3'-Benzyl-4-bromo-5'-(4-phenylpiperazin-1-yl)[1,1':2',1"-terphenyl]-4'-carbonitrile (11e)

White solid; yield: $467 \mathrm{mg}$ (0.80 mmol, $80 \%) ; \mathrm{mp} 170-172{ }^{\circ} \mathrm{C} ; R_{f}=0.5$ (EtOAc-hexane 1:49).

IR (ATR): $2212 \mathrm{~cm}^{-1}(\mathrm{C} \equiv \mathrm{N})$.

${ }^{1} \mathrm{H} \mathrm{NMR}\left(400 \mathrm{MHz}, \mathrm{CDCl}_{3}\right): \delta=3.31-3.37\left(\mathrm{~m}, 8 \mathrm{H}, 4 \times \mathrm{NCH}_{2}\right), 4.06(\mathrm{~s}, 2$ $\left.\mathrm{H}, \mathrm{CH}_{2}\right), 6.70-6.83(\mathrm{~m}, 7 \mathrm{H}, \mathrm{ArH}), 6.86(\mathrm{~s}, 1 \mathrm{H}, \mathrm{ArH}), 6.89(\mathrm{~d}, J=8.0 \mathrm{~Hz}$, $2 \mathrm{H}, \mathrm{ArH}), 6.99-7.09$ (m, 6 H, ArH), 7.12-7.23 (m, 4 H, ArH).
${ }^{13} \mathrm{C}$ NMR $\left(100 \mathrm{MHz}, \mathrm{CDCl}_{3}\right): \delta=38.5,49.6,51.9,107.6,116.5,117.8$, 118.7, 120.2, 121.5, 126.1, 127.2, 128.0, 128.2, 128.5, 129.2, 130.7, 130.9, 131.0, 135.8, 137.7, 139.2, 139.7, 145.0, 146.0, 151.2, 155.4 .

GC-MS: $m / z=585[\mathrm{M}+1]^{+}, 586[\mathrm{M}+2]^{+}$.

Anal. Calcd for $\mathrm{C}_{36} \mathrm{H}_{30} \mathrm{BrN}_{3}$ : C, 73.97; $\mathrm{H}, 5.17 ; \mathrm{N}, 7.19$. Found: C, 74.01; H, 5.36; N, 6.99.

\section{3'-Benzyl-4-methyl-5'-(piperidin-1-yl)[1,1':2',1"-terphenyl]-4'- carbonitrile (11f)}

White solid; yield: $419 \mathrm{mg}$ (0.95 mmol, 95\%); $\mathrm{mp} 156-158^{\circ} \mathrm{C} ; R_{f}=0.5$ (EtOAc-hexane 1:49).

IR (ATR): $2210 \mathrm{~cm}^{-1}(\mathrm{C} \equiv \mathrm{N})$.

${ }^{1} \mathrm{H}$ NMR $\left(400 \mathrm{MHz}, \mathrm{CDCl}_{3}\right): \delta=1.47-1.57\left(\mathrm{~m}, 2 \mathrm{H}, \mathrm{CH}_{2}\right), 1.68-1.77(\mathrm{~m}$, $\left.4 \mathrm{H}, 2 \times \mathrm{CH}_{2}\right), 2.15\left(\mathrm{~s}, 3 \mathrm{H}, \mathrm{CH}_{3}\right), 3.12\left(\mathrm{t}, J=5.2 \mathrm{~Hz}, 4 \mathrm{H}, 2 \times \mathrm{NCH}_{2}\right), 4.04$ $\left(\mathrm{s}, 2 \mathrm{H}, \mathrm{CH}_{2}\right), 6.76(\mathrm{t}, J=8.4 \mathrm{~Hz}, 4 \mathrm{H}, \mathrm{ArH}), 6.80-6.87(\mathrm{~m}, 5 \mathrm{H}, \mathrm{ArH})$, 6.97-7.10 (m, $6 \mathrm{H}, \mathrm{ArH})$.

${ }^{13} \mathrm{C}$ NMR $\left(100 \mathrm{MHz}, \mathrm{CDCl}_{3}\right): \delta=21.1,24.1,26.2,38.4,53.6,106.9$, $118.2,119.0,125.9,126.7,127.7,128.1,128.4,128.5,129.3,130.9$, 135.0, 136.6, 138.1, 138.4, 139.5, 144.3, 147.1, 156.8 .

GC-MS: $m / z=443[\mathrm{M}+1]^{+}$.

Anal. Calcd for $\mathrm{C}_{32} \mathrm{H}_{30} \mathrm{~N}_{2}$ : C, 86.84; H, 6.83; N, 6.33. Found: C, 86.77; $\mathrm{H}$, $6.82 ; \mathrm{N}, 6.24$.

\section{3'-Benzyl-4-methoxy-5'-(piperidin-1-yl)[1,1':2',1"-terphenyl]-4'- carbonitrile (11g)}

White solid; yield: $421 \mathrm{mg}$ (0.92 mmol, 92\%); mp 95-97 ${ }^{\circ} \mathrm{C} ; R_{\mathrm{f}}=0.5$ (EtOAc-hexane 1:49).

IR (ATR): $2212 \mathrm{~cm}^{-1}(\mathrm{C} \equiv \mathrm{N})$.

${ }^{1} \mathrm{H}$ NMR $\left(400 \mathrm{MHz}, \mathrm{CDCl}_{3}\right): \delta=1.48-1.59\left(\mathrm{~m}, 2 \mathrm{H}, \mathrm{CH}_{2}\right), 1.68-1.77(\mathrm{~m}$, $\left.4 \mathrm{H}, 2 \times \mathrm{CH}_{2}\right), 3.12\left(\mathrm{t}, J=5.2 \mathrm{~Hz}, 4 \mathrm{H}, 2 \times \mathrm{NCH}_{2}\right), 3.63\left(\mathrm{~s}, 3 \mathrm{H}, \mathrm{OCH}_{3}\right)$, $4.04\left(\mathrm{~s}, 2 \mathrm{H}, \mathrm{CH}_{2}\right), 6.57$ (d, J = 8.4 Hz, $\left.2 \mathrm{H}, \mathrm{ArH}\right), 6.76(\mathrm{t}, J=8.4 \mathrm{~Hz}, 4 \mathrm{H}$, ArH), 6.82-6.88 (m, $3 \mathrm{H}, \mathrm{ArH}), 6.98-7.10$ (m, $6 \mathrm{H}, \mathrm{ArH})$.

${ }^{13} \mathrm{C}$ NMR $\left(100 \mathrm{MHz}, \mathrm{CDCl}_{3}\right): \delta=24.2,26.2,38.5,53.6,55.1,106.8$, $113.1,118.3,119.0,125.9,126.8,127.8,128.1,128.5,130.6,130.9$, $133.4,135.0,138.4,139.5,144.3,146.7,156.8,158.5$.

GC-MS: $m / z=459[\mathrm{M}+1]^{+}$.

Anal. Calcd for $\mathrm{C}_{32} \mathrm{H}_{30} \mathrm{~N}_{2} \mathrm{O}$ : C, 83.81; H, 6.59; N, 6.11. Found: C, 83.08; H, 6.70; N, 6.07.

\section{3'-Benzyl-4-methoxy-5'-(4-phenylpiperazin-1-yl)[1,1':2',1"-ter- phenyl]-4'-carbonitrile (11h)}

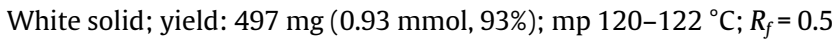
(EtOAc-hexane 1:49).

IR (ATR): $2209 \mathrm{~cm}^{-1}(\mathrm{C} \equiv \mathrm{N})$.

$\left.{ }^{1} \mathrm{H} \mathrm{NMR} \mathrm{(400} \mathrm{MHz,} \mathrm{CDCl}_{3}\right): \delta=3.32-3.38\left(\mathrm{~m}, 8 \mathrm{H}, 4 \times \mathrm{NCH}_{2}\right), 3.64(\mathrm{~s}, 3$ $\left.\mathrm{H}, \mathrm{OCH}_{3}\right), 4.07\left(\mathrm{~s}, 2 \mathrm{H}, \mathrm{CH}_{2}\right), 6.58(\mathrm{~d}, J=8.8 \mathrm{~Hz}, 2 \mathrm{H}, \mathrm{ArH}), 6.77(\mathrm{t}, J=7.6$ $\mathrm{Hz}, 4 \mathrm{H}, \mathrm{ArH}), 6.80-6.94$ (m, $6 \mathrm{H}, \mathrm{ArH}), 6.98-7.11$ (m, $6 \mathrm{H}, \mathrm{ArH}), 7.21$ (t, $J=8.8 \mathrm{~Hz}, 2 \mathrm{H}, \mathrm{ArH})$.

${ }^{13} \mathrm{C}$ NMR $\left(100 \mathrm{MHz}, \mathrm{CDCl}_{3}\right): \delta=38.5,49.6,52.0,55.2,106.9,113.2$, $116.5,118.1,118.9,120.2,126.0,126.9,127.9,128.1,128.5,129.2$, $130.6,130.8,133.2,135.9,138.2,139.4,144.7,147.0,151.2,155.3$, 158.6 .

GC-MS: $m / z=536[\mathrm{M}+1]^{+}$.

Anal. Calcd for $\mathrm{C}_{37} \mathrm{H}_{33} \mathrm{~N}_{3} \mathrm{O}$ : C, 82.96; H, 6.21; N, 7.84. Found: C, 82.86; H, 6.25; N, 7.73. 


\section{2-Benzyl-6-(naphthalen-2-yl)-4-(piperidin-1-yl)[1,1'-biphenyl]-3- carbonitrile (11i)}

White solid; yield: $430 \mathrm{mg}$ (0.90 mmol, 90\%); $\mathrm{mp} 182-184^{\circ} \mathrm{C} ; R_{f}=0.5$ (EtOAc-hexane 1:49).

IR (ATR): $2214 \mathrm{~cm}^{-1}(\mathrm{C} \equiv \mathrm{N})$.

${ }^{1} \mathrm{H} \mathrm{NMR}\left(400 \mathrm{MHz}, \mathrm{CDCl}_{3}\right): \delta=1.63-1.71\left(\mathrm{~m}, 2 \mathrm{H}, \mathrm{CH}_{2}\right), 1.82-1.90(\mathrm{~m}$, $\left.4 \mathrm{H}, 2 \times \mathrm{CH}_{2}\right), 3.28\left(\mathrm{t}, J=5.2 \mathrm{~Hz}, 4 \mathrm{H}, 2 \times \mathrm{NCH}_{2}\right), 4.22\left(\mathrm{~s}, 2 \mathrm{H}, \mathrm{CH}_{2}\right), 6.90-$ $6.96(\mathrm{~m}, 4 \mathrm{H}, \mathrm{ArH}), 7.08-7.13(\mathrm{~m}, 5 \mathrm{H}, \mathrm{ArH}), 7.14-7.23(\mathrm{~m}, 3 \mathrm{H}, \mathrm{ArH})$, 7.42-7.50 (m, $2 \mathrm{H}, \operatorname{ArH}), 7.56(\mathrm{~d}, J=8.4 \mathrm{~Hz}, 1 \mathrm{H}, \operatorname{ArH}), 7.64(\mathrm{~s}, 1 \mathrm{H}$, ArH), 7.71-7.78 (m, $2 \mathrm{H}, \mathrm{ArH})$.

${ }^{13} \mathrm{C}$ NMR $\left(100 \mathrm{MHz}, \mathrm{CDCl}_{3}\right): \delta=24.2,26.2,38.4,53.6,107.3,118.2$, 119.3, 125.9, 126.1, 126.2, 126.8, 126.9, 127.4, 127.6, 127.8, 127.9, $128.1,128.4,128.5,130.9,132.1,132.9,135.1,138.2,138.8,139.5$, 144.5, 147.0, 156.9.

GC-MS: $m / z=479[\mathrm{M}+1]^{+}$.

Anal. Calcd for $\mathrm{C}_{35} \mathrm{H}_{30} \mathrm{~N}_{2}$ : C, 87.83; $\mathrm{H}, 6.32 ; \mathrm{N}, 5.85$. Found: C, 83.69; H, $6.36 ; \mathrm{N}, 5.58$.

3'-Benzyl-6'-methyl-5'-(4-phenylpiperazin-1-yl)[1,1':2',1"-terphenyl]-4'-carbonitrile (11j)

White solid; yield: $384 \mathrm{mg}(0.74 \mathrm{mmol}, 74 \%) ; \mathrm{mp} 178-180{ }^{\circ} \mathrm{C} ; R_{f}=0.5$ (EtOAc-hexane 1:49).

IR (ATR): $2217 \mathrm{~cm}^{-1}(\mathrm{C} \equiv \mathrm{N})$.

${ }^{1} \mathrm{H} \mathrm{NMR}\left(400 \mathrm{MHz}, \mathrm{CDCl}_{3}\right): \delta=1.99\left(\mathrm{~s}, 3 \mathrm{H}, \mathrm{CH}_{3}\right), 3.22-3.60(\mathrm{~m}, 8 \mathrm{H}$, $\left.4 \times \mathrm{NCH}_{2}\right), 3.98\left(\mathrm{~s}, 2 \mathrm{H}, \mathrm{CH}_{2}\right), 6.64\left(\mathrm{dd}, J_{1}=7.2 \mathrm{~Hz}, J_{2}=1.6 \mathrm{~Hz}, 2 \mathrm{H}, \mathrm{ArH}\right)$, $6.78\left(\mathrm{dt}, J_{1}=7.2 \mathrm{~Hz}, J_{2}=1.6 \mathrm{~Hz}, 5 \mathrm{H}, \mathrm{ArH}\right), 6.89-6.96(\mathrm{~m}, 5 \mathrm{H}, \mathrm{ArH})$, 6.95-7.09 (m, $6 \mathrm{H}, \mathrm{ArH}), 7.20(\mathrm{t}, J=8.4 \mathrm{~Hz}, 2 \mathrm{H}, \mathrm{ArH})$.

${ }^{13} \mathrm{C}$ NMR $\left(100 \mathrm{MHz}, \mathrm{CDCl}_{3}\right): \delta=17.2,38.2,50.5,50.7,111.4,116.6$, 118.5, 120.0, 126.0, 126.6, 127.4, 127.7, 128.1, 128.5, 129.1, 129.3, 130.3, 133.9, 138.6, 139.5, 139.6, 139.9, 141.4, 148.2, 151.8, 152.8.

GC-MS: $m / z=520[\mathrm{M}+1]^{+}$.

Anal. Calcd for $\mathrm{C}_{37} \mathrm{H}_{33} \mathrm{~N}_{3}$ : C, 85.51; $\mathrm{H}, 6.40 ; \mathrm{N}, 8.09$. Found: C, 85.38; H, $6.70 ; \mathrm{N}, 8.07$.

\section{3'-Benzyl-6'-methyl-5'-(piperidin-1-yl)[1,1':2',1"-terphenyl]-4'- carbonitrile (111)}

White solid; yield: $349 \mathrm{mg}$ (0.79 mmol, 79\%); $\mathrm{mp} 156-158^{\circ} \mathrm{C} ; R_{f}=0.5$ (EtOAc-hexane 1:49).

IR (ATR): $2213 \mathrm{~cm}^{-1}(\mathrm{C} \equiv \mathrm{N})$.

${ }^{1} \mathrm{H}$ NMR (400 MHz, $\left.\mathrm{CDCl}_{3}\right): \delta=1.55-1.74\left(\mathrm{~m}, 6 \mathrm{H}, 3 \times \mathrm{CH}_{2}\right), 1.94(\mathrm{~s}, 3$ $\left.\mathrm{H}, \mathrm{CH}_{3}\right), 3.10-3.36\left(\mathrm{~m}, 4 \mathrm{H}, 2 \times \mathrm{NCH}_{2}\right), 3.96\left(\mathrm{~s}, 2 \mathrm{H}, \mathrm{CH}_{2}\right), 6.63\left(\mathrm{dd}, J_{1}=\right.$ $\left.7.6 \mathrm{~Hz}, J_{2}=2.0 \mathrm{~Hz}, 2 \mathrm{H}, \mathrm{ArH}\right), 6.74-6.80(\mathrm{~m}, 4 \mathrm{H}, \mathrm{ArH}), 6.86-6.93(\mathrm{~m}, 3$ $\mathrm{H}, \mathrm{ArH}), 6.94-7.10(\mathrm{~m}, 6 \mathrm{H}, \mathrm{ArH})$.

${ }^{13} \mathrm{C}$ NMR $\left(100 \mathrm{MHz}, \mathrm{CDCl}_{3}\right): \delta=16.9,24.3,26.9,38.1,52.0,110.5$, $118.8,125.9,126.4,126.5,127.3,127.6,128.0,128.5,129.4,130.3$, 133.8, 138.7, 138.8, 139.7, 140.2, 141.0, 148.0, 154.5.

GC-MS: $m / z=443[\mathrm{M}+1]^{+}$.

Anal. Calcd for $\mathrm{C}_{32} \mathrm{H}_{30} \mathrm{~N}_{2}$ : C, 86.84; $\mathrm{H}, 6.83 ; \mathrm{N}, 6.33$. Found: C, 86.62; H, $6.87 ; \mathrm{N}, 6.11$.

\section{3'-Benzyl-5'-(methylthio)[1,1':2',1"-terphenyl]-4'-carbonitrile (12a)}

A mixture of 4-(methylthio)-2-oxo-6-phenyl-2H-pyran-3-carbonitrile (5a; $243 \mathrm{mg}, 1.0 \mathrm{mmol}, 1.0$ equiv), 1,3-diphenylacetone (10; 0.24 $\mathrm{mL}, 1.2 \mathrm{mmol}, 1.2$ equiv), and powdered $\mathrm{KOH}(84 \mathrm{mg}, 1.5 \mathrm{mmol}, 1.5$ equiv) in DMF ( $5 \mathrm{~mL}$ ) was stirred at r.t. for $12 \mathrm{~h}$. The course of the reaction was monitored by TLC. On completion of the reaction, few ice pieces were added to the reaction mixture and neutralized with aq 2 $\mathrm{M} \mathrm{HCl}$. After that the reaction mixture was extracted with EtOAc $(3 \times$ $10 \mathrm{~mL}$ ). The combined organic layers were dried (anhyd $\mathrm{Na}_{2} \mathrm{SO}_{4}$ ), filtered, and, concentrated under vacuum. The crude product was purified by neutral alumina column chromatography using EtOAc-hexane (1:49) as an eluent to afford 12a; white solid; yield: $242 \mathrm{mg}(0.62$ $\mathrm{mmol}, 62 \%) ; \mathrm{mp} 144-146{ }^{\circ} \mathrm{C} ; R_{f}=0.4$ (EtOAc-hexane 1:49).

IR (ATR): $2211 \mathrm{~cm}^{-1}(\mathrm{C} \equiv \mathrm{N})$.

${ }^{1} \mathrm{H} \mathrm{NMR}\left(400 \mathrm{MHz}, \mathrm{CDCl}_{3}\right): \delta=2.49\left(\mathrm{~s}, 3 \mathrm{H}, \mathrm{SCH}_{3}\right), 4.07\left(\mathrm{~s}, 2 \mathrm{H}, \mathrm{CH}_{2}\right)$, 6.72-6.77 (m, 4 H, ArH), 6.90-6.96 (m, 2 H, ArH), 6.98-7.09 (m, 9 H, $\operatorname{ArH}), 7.12$ (s, $1 \mathrm{H}, \mathrm{ArH})$.

${ }^{13} \mathrm{C}$ NMR $\left(100 \mathrm{MHz}, \mathrm{CDCl}_{3}\right): \delta=14.7,37.3,110.7,115.6,124.4,125.1$, $126.1,126.2,126.7,126.8,127.1,127.4,128.3,129.5,136.5,137.7$, $137.8,139.3,142.2,143.1,145.6$.

GC-MS: $m / z=392[\mathrm{M}+1]^{+}, 393[\mathrm{M}+2]^{+}$.

Anal. Calcd for $\mathrm{C}_{27} \mathrm{H}_{21} \mathrm{NS}$ : C, 82.83; H, 5.41; N, 3.58; S, 8.19. Found: C, $82.21 ; \mathrm{H}, 5.52 ; \mathrm{N}, 3.42 ; \mathrm{S}, 8.09$.

\section{Acknowledgment}

Fateh V. Singh is thankful to the SAIF, VIT University, Vellore Campus for providing spectroscopic analysis. Priyanka B. Kole is grateful to VIT Chennai Campus for providing a fellowship.

\section{Supporting Information}

Supporting information for this article is available online at https://doi.org/10.1055/s-0037-1610332.

\section{References}

(1) (a) Canel, C.; Moraes, R. M.; Dayan, F. E.; Ferreira, D. Photochemistry 2000, 54, 115. (b) Tsutsui, C.; Yamada, Y.; Ando, M.; Toyama, D.; Wu, J.-l.; Wang, L.; Taketani, S.; Kataoka, T. Bioorg. Med. Chem. Lett. 2009, 19, 4084.

(2) (a) Mondal, S.; Panda, G. RSC Adv. 2014, 4, 28317. (b) Wood, P. M.; Woo, L. W. L.; Labrosse, J.-R.; Trusselle, M. N.; Abbate, S.; Longhi, G.; Castiglioni, E.; Lebon, F.; Purohit, A.; Reed, M. J.; Potter, B. V. L. J. Med. Chem. 2008, 51, 4226.

(3) Yoshihara, H. A. I.; Apriletti, J. W.; Baxter, J. D.; Scanlan, T. S. Bioorg. Med. Chem. Lett. 2001, 11, 2821.

(4) Kong, X.; Chen, L.; Jiao, L.; Lian, F.; Lu, J.; Zhu, K.; Du, D.; Liu, J.; Ding, H.; Zhang, N.; Shen, J.; Zheng, M.; Chen, K.; Liu, X.; Jiang, H.; Luo, C. J. Med. Chem. 2014, 57, 9512; and the references cited therein.

(5) (a) Wai, J. S.; Egbertson, M. S.; Payne, L. S.; Fisher, T. E.; Embrey, M. W.; Tran, L. O.; Melamed, M. W.; Langford, H. M.; Guare, J. P.; Zhuang, L.; Grey, V. E.; Vacca, J. P.; Holloway, M. K.; NaylorOlsen, A. M.; Hazuda, D. J.; Felock, P. J.; Wolfe, A. L.; Stillmock, K. A.; Schleif, W. A.; Gabryelski, L. J.; Young, S. D. J. Med. Chem. 2000, 43, 4924. (b) Zhuang, L.; Wai, J. S.; Embrey, M. W.; Fisher, T. E.; Egbertson, M. S.; Payne, L. S.; Guare, J. P.; Vacca, J. P.; Hazuda, D. J.; Felock, P. J.; Wolfe, A. L.; Moyer, G.; Schlelf, W. A.; Gabryelski, L. J.; Leonard, Y. M.; Lynch, J. J.; Michelson, S. R.; Young, S. D. J. Med. Chem. 2003, 46, 453. (c) Long, Y.-Q.; Jiang, X.H.; Dayam, R.; Sanchez, T.; Shoemaker, R.; Sel, S.; Neamati, N. J. Med. Chem. 2004, 47, 2561. (d) Cheltsov, A. V.; Aoyagi, M.; 
Aleshin, A.; Yu, C.-W. E.; Gilliland, T.; Zhai, D.; Bobkov, A. A.; Reed, J. C.; Liddington, R. C.; Abagyan, R. J. Med. Chem. 2010, 53, 3899.

(6) (a) Procopiou, P. A.; Browning, C.; Buckley, J. M.; Clark, K. L.; Fechner, L.; Gore, P. M.; Hancock, A. P.; Hodgson, S. T.; Holmes, D. S.; Kranz, M.; Looker, B. E.; Morriss, K. M. L.; Parton, D. L.; Russell, L. J.; Slack, R. J.; Sollis, S. L.; Vile, S.; Watts, C. J. J. Med. Chem. 2011, 54, 2183. (b) Procopiou, P. A.; Ford, A. J.; Gore, P. M.; Looker, B. E.; Hodgson, S. T.; Holmes, D. S.; Vile, S.; Clark, K. L.; Saunders, K. A.; Slack, R. J.; Rowedder, J. E.; Watts, C. J. ACS Med. Chem. Lett. 2017, 8, 577.

(7) (a) Ohtake, Y.; Sato, T.; Kobayashi, T.; Nishimoto, M.; Taka, N.; Takano, K.; Yamamoto, K.; Ohmori, M.; Yamaguchi, M.; Takami, K.; Yeu, S.-Y.; Ahn, K.-H.; Matsuoka, H.; Morikawa, K.; Suzuki, M.; Hagita, H.; Ozawa, K.; Yamaguchi, K.; Kato, M.; Ikeda, S. J. Med. Chem. 2012, 55, 7828. (b) Li, Y.; Shi, Z.; Chen, L.; Zheng, S.; Li, S.; Xu, B.; Liu, Z.; Liu, J.; Deng, C.; Ye, F. J. Med. Chem. 2017, 60.4173.

(8) Mahesh, S.; Anand, R. V. Org. Biomol. Chem. 2017, 15, 8393.

(9) (a) Novelli, A.; Rosi, E. J. Chemother. 2017, 29, 10. (b) Wang, S.; Yin, Y.; Wang, J. Appl. Microbiol. Biotechnol. 2018, 102, 1997.

(10) (a) Takamatsu, S.; Hodges, T. W.; Rajbhandari, I.; Gerwick, W. H.; Hamann, M. T.; Nagle, D. G. J. Nat. Prod. 2003, 66, 605. (b) Wegener, A.; Miller, K. A. J. Org. Chem. 2017, 82, 11655; and references cited therein.

(11) (a) Jablonicka, V.; Ziegler, J.; Vatehova, Z.; Liskova, D.; Heilmann, I.; Oblozinsky, M.; Heilmann, M. J. Plant Physiol. 2018, 223, 1. (b) Yang, X.; Tsui, G. C. Org. Lett. 2018, 20, 1179.

(12) Kern, L.; Xu, R.; Julien, S.; Suter, D. M.; Preynat-Seauve, O.; Baquie, M.; Poncet, A.; Combescure, C.; Stoppini, L.; Thriel, C. V.; Krause, K.-H. Curr. Med. Chem. 2013, 20, 710.

(13) Kito, Y.; Suzuki, H.; Edwards, F. R. J. Smooth Muscle Res. 2002, 38, 165.

(14) Xu, M.-M.; Wang, H.-Q.; Wan, Y.; He, G.; Yan, J.; Zhang, S.; Wang, S.-L.; Shi, F. Org. Chem. Front. 2017, 4, 358.

(15) Liang, F.; Ji, X.; Zhang, J.; Cao, S. Org. Chem. Front. 2016, 3, 1425.

(16) (a) Chowdhury, S.; Georghiou, P. E. Tetrahedron Lett. 1999, 40, 7599. (b) Nobre, S. M.; Monteiro, A. L. Tetrahedron Lett. 2004, 45, 8225. (c) Liegault, B.; Renaud, J.-L.; Bruneau, C. Chem. Soc. Rev. 2008, 37, 290. (d) Zhao, G.; Zhang, K.; Wang, L.; Li, J.; Zou, D.; $\mathrm{Wu}, \mathrm{Y}$.; Wu, Y. Tetrahedron Lett. 2015, 56, 6700.

(17) Xu, W.; Paira, R.; Yoshikai, N. Org. Lett. 2015, 17, 4192.

(18) Zhang, J.; Lu, G.; Sun, H.; Shen, Q. Org. Lett. 2016, 18, 2860.

(19) Mahesh, S.; Kant, G.; Anand, R. V. RSC Adv. 2016, 6, 80718.
(20) Hemelaere, R.; Champagne, P. A.; Desroches, J.; Paquin, J.-F. J. Fluorine Chem. 2016, 190, 1.

(21) Ueda, M.; Nakakoji, D.; Kuwahara, Y.; Nishimura, K.; Ryu, I. Tetrahedron Lett. 2016, 57, 4142.

(22) (a) Goal, A.; Singh, F. V.; Dixit, M.; Verma, D.; Raghunandan, R.; Maulik, P. R. Chem. Asian. J. 2007, 2, 239. (b) Singh, F. V.; Chaurasia, S.; Joshi, M. D.; Srivastava, A. K.; Goel, A. Bioorg. Med. Chem. Lett. 2007, 17, 2425. (c) Goal, A.; Ram, V. J. Tetrahedron 2009, 65, 7865.

(23) (a) Singh, F. V.; Kumar, V.; Goal, A. Synlett 2007, 13, 2086. (b) Singh, F. V.; Kumar, V.; Kumar, B.; Goal, A. Tetrahedron 2007, 63, 10971. (c) Goal, A.; Singh, F. V.; Kumar, V.; Reichert, M.; Gulder, T. A. M.; Bringmann, G. J. Org. Chem. 2007, 72, 7765. (d) Kumar, A.; Singh, F. V.; Goal, A. Tetrahedron Lett. 2007, 48, 8223. (e) Goel, A.; Kumar, V.; Hemberger, Y.; Singh, F. V.; Nag, P.; Knauer, M.; Kant, R.; Raghunandan, R.; Maulik, P. R.; Bringmann, G. J. Org. Chem. 2016, 81, 10721.

(24) (a) Goal, A.; Singh, F. V.; Sharon, A.; Maulik, P. R. Synlett 2005, 623. (b) Goal, A.; Singh, F. V.; Verma, D. Synlett 2005, 2027. (c) Goel, A.; Taneja, G.; Raghuvanshi, A.; Kant, R.; Maulik, P. R. Org. Biomol. Chem. 2013, 11, 5239.

(25) (a) Goel, A.; Singh, S. P.; Kumar, A.; Kant, R.; Maulik, P. R. Org. Lett. 2009, 11, 5122. (b) Goel, A.; Kumar, V.; Chaurasia, S.; Rawat, M.; Prasad, R.; Anand, R. S. J. Org. Chem. 2010, 75, 3656. (c) Maurya, H. K.; Tandon, V. K.; Kumar, B.; Kumar, A.; Huch, V.; Ram, V. J. Org. Biomol. Chem. 2012, 10, 605. (d) Goel, A.; Kumar, V.; Singh, S. P.; Sharma, A.; Prakash, S.; Singh, C.; Anand, R. S. J. Mater. Chem. 2012, 22, 14880. (e) Goel, A.; Sharma, A.; Kathuria, M.; Bhattacharjee, A.; Verma, A.; Mishra, P. R.; Nazir, A.; Mitra, K. Org. Lett. 2014, 16, 756. (f) Goel, A.; Umar, S.; Nag, P.; Sharma, A.; Kumar, L.; Shamsuzzama Hossain, Z.; Gayen, J. R.; Nazir, A. Chem. Commun. 2015, 51, 5001. (g) Sharma, A.; Umar, S.; Kar, P.; Singh, K.; Sachdev, M.; Goel, A. Analyst 2016, 141, 137. (h) Taneja, G.; Gupta, C. P.; Mishra, S.; Srivastava, R.; Rahuja, N.; Rawat, A. K.; Pandey, J.; Gupta, A. P.; Jaiswal, N.; Jiaur, G. R.; Tamrakar, A. K.; Srivastava, A. K.; Goel, A. Med. Chem. Commun. 2017, 8, 329.

(26) Shetgaonkar, S. E.; Singh, F. V. Synthesis 2018, 50, 3540.

(27) (a) Goel, A.; Singh, F. V. Tetrahedron Lett. 2005, 46, 5585. (b) Goel, A.; Verma, D.; Singh, F. V. Tetrahedron Lett. 2005, 46, 8487. (c) Singh, F. V.; Kumar, A.; Goel, A. Tetrahedron Lett. 2006, 47, 7767. (d) Kumar, V.; Singh, F. V.; Parihar, A.; Goel, A. Tetrahedron Lett. 2009, 50, 680. (e) Goel, A.; Kumar, V.; Nag, P.; Bajpai, V.; Kumar, B.; Singh, C.; Prakash, S.; Anand, R. S. J. Org. Chem. 2011, 76, 7474 . 